\title{
Excited-state Intramolecular Proton Transfer Reaction and Ground-State Hole Dynamics of 4'- $N, N$-Dialkylamino-3-Hydroxyflavone in Ionic Liquids Studied by Transient Absorption Spectroscopy
}

Hanamichi Miyabayashi,${ }^{+*}$ Kaori Fujii, ${ }^{+}$Takumi Watanabe,,+ Yoshihiro Matano, ${ }^{\not}$

Takatsugu Endo, ${ }^{\S}$ and Yoshifumi Kimurat, ${ }^{*}$

${ }^{\dagger}$ Department of Applied Chemistry, Graduate School of Science and Engineering, Doshisha University, Kyotanabe, 610-0321 Kyoto, Japan

Department of Chemistry, Graduate School of Science and Technology, Niigata University, Nishi-ku, 950-2181 Niigata, Japan.

${ }^{\S}$ Department of Molecular Chemistry and Biochemistry, Faculty of Science and Engineering, Doshisha University, Kyotanabe, 610-0321 Kyoto, Japan

*Kimura, Y.: yokimura@mail.doshisha.ac.jp

*Miyabayashi, H.: hanamichi19950825@gmail.com 


\section{S1. Procedure of the spectra fitting using five log-normal functions.}

In practice, the transient absorption spectrum was simulated using Eq. (5) and the following method. First, a spectrum obtained at a long delay time (250 ps) was simulated because the band shape in this spectrum hardly varied with time. In the fitting, the $v_{P, i}, \Delta_{i}$, and $\gamma_{i}$ parameters for the GSB and two IEs were obtained from simulations of the ground-state absorption and steady-state emission spectra. By fixing these parameters, other parameters such as $h_{i}$ for all species and $v_{P, i}, \Delta_{i}$, and $\gamma_{i}$ for the $\mathrm{N}^{*}$ and $\mathrm{T}^{*}$ bands were obtained by fitting. Using these values as the initial guess, the spectra at shorter delay times were fitted successively using the parameters obtained at longer delay times as the initial guess. Once the spectra at all delay times had been simulated, we inspected the parameters. The band shapes derived from the IE and the ESA of $\mathrm{T}^{*}$ did not vary significantly though the peak position of the ESA of T* apparently shifts driven by the shift of the neighboring component, IE of $\mathrm{N}^{*}$. The $v_{P, i}, \Delta_{i}$, and $\gamma_{i}$ parameters for these bands were fixed to the averaged values obtained for delay times between 100 and 300 ps. The bands assigned to the IE and the ESA of $N^{*}$ showed large shifts with time, as was observed in the case of the fluorescence spectrum of $\mathrm{N}^{*}$. Thus, we fixed $\gamma_{i}$ alone to the averaged values obtained at delay times between 100 and 300 ps. Further, because we wanted to obtain the ground-state hole dynamics, we fixed only the $\gamma_{i}$ parameter of the GSB to that obtained from the absorption spectrum. Then, the spectra for all time delays were fitted again by fixing the parameters mentioned above. 
Table S1. Absorption band center of the ground state and the peak positions of the fluorescence excited at $400 \mathrm{~nm}$.

\begin{tabular}{cccccc}
\hline $\mathrm{C}_{\mathrm{n}} \mathrm{HF}$ & $\mathrm{Cation}$ & $v_{a b s} / \mathrm{nm}$ & $v_{N^{*}} / \mathrm{nm}$ & $v_{T^{*}} / \mathrm{nm}$ & $K^{*}{ }_{\text {eq }}$ \\
\hline $\mathrm{C}_{2} \mathrm{HF}$ & {$\left[\mathrm{C}_{2} \mathrm{mim}\right]^{+}$} & 410 & 535 & 590 & 0.43 \\
$\mathrm{C}_{2} \mathrm{HF}$ & {$\left[\mathrm{C}_{8} \mathrm{mim}\right]^{+}$} & 414 & 527 & 584 & 0.52 \\
$\mathrm{C}_{2} \mathrm{HF}$ & {$\left[\mathrm{P}_{4441}\right]^{+}$} & 414 & 521 & 582 & 0.60 \\
$\mathrm{C}_{2} \mathrm{HF}$ & {$\left[\mathrm{P}_{4448}\right]^{+}$} & 415 & 515 & 581 & 1.17 \\
$\mathrm{C}_{2} \mathrm{HF}$ & {$\left[\mathrm{P}_{8881}\right]^{+}$} & 415 & 502 & 580 & 1.31 \\
$\mathrm{C}_{2} \mathrm{HF}$ & {$\left[\mathrm{P}_{66614}\right]^{+}$} & 415 & 498 & 579 & 1.72 \\
$\mathrm{C}_{2} \mathrm{HF}$ & {$\left[\mathrm{P}_{88816}\right]^{+}$} & 416 & 491 & 578 & 2.09 \\
$\mathrm{C}_{4} \mathrm{HF}$ & {$\left[\mathrm{C}_{2} \mathrm{mim}\right]^{+}$} & 412 & 539 & 593 & 0.30 \\
$\mathrm{C}_{4} \mathrm{HF}$ & {$\left[\mathrm{C}_{8} \mathrm{mim}\right]^{+}$} & 416 & 529 & 586 & 0.34 \\
$\mathrm{C}_{4} \mathrm{HF}$ & {$\left[\mathrm{P}_{4441}\right]^{+}$} & 415 & 527 & 584 & 0.45 \\
$\mathrm{C}_{4} \mathrm{HF}$ & {$\left[\mathrm{P}_{4448}\right]^{+}$} & 415 & 513 & 583 & 1.06 \\
$\mathrm{C}_{4} \mathrm{HF}$ & {$\left[\mathrm{P}_{8881}\right]^{+}$} & 417 & 507 & 582 & 1.22 \\
$\mathrm{C}_{4} \mathrm{HF}$ & {$\left[\mathrm{P}_{66614}\right]^{+}$} & 416 & 498 & 581 & 1.67 \\
$\mathrm{C}_{4} \mathrm{HF}$ & {$\left[\mathrm{P}_{88816}\right]^{+}$} & 417 & 493 & 579 & 1.92 \\
$\mathrm{C}_{8} \mathrm{HF}$ & {$\left[\mathrm{C}_{2} \mathrm{mim}\right]^{+}$} & 412 & 540 & 593 & 0.29 \\
$\mathrm{C}_{8} \mathrm{HF}$ & {$\left[\mathrm{C}_{8} \mathrm{mim}\right]^{+}$} & 415 & 522 & 586 & 0.41 \\
$\mathrm{C}_{8} \mathrm{HF}$ & {$\left[\mathrm{P}_{4441}\right]^{+}$} & 416 & 528 & 585 & 0.44 \\
$\mathrm{C}_{8} \mathrm{HF}$ & {$\left[\mathrm{P}_{4448}\right]^{+}$} & 416 & 512 & 582 & 1.02 \\
$\mathrm{C}_{8} \mathrm{HF}$ & {$\left[\mathrm{P}_{8881}\right]^{+}$} & 417 & 502 & 581 & 1.15 \\
$\mathrm{C}_{8} \mathrm{HF}$ & {$\left[\mathrm{P}_{66614}\right]^{+}$} & 418 & 510 & 580 & 1.21 \\
$\mathrm{C}_{8} \mathrm{HF}$ & {$\left[\mathrm{P}_{88816}\right]^{+}$} & 418 & 502 & 579 & 1.67 \\
\hline & & & & &
\end{tabular}


Table S2. Parameters used for the spectral decomposition of the fluorescence obtained at $400 \mathrm{~nm}$ excitation.

\begin{tabular}{ccccccc}
\hline $\mathrm{C}_{\mathrm{n}} \mathrm{HF}$ & Cation & $h_{T} / h_{N}$ & $\gamma_{N}$ & $\gamma_{T}$ & $\Delta_{N}$ & $\Delta_{T}$ \\
\hline $\mathrm{C}_{2} \mathrm{HF}$ & {$\left[\mathrm{C}_{2} \mathrm{mim}\right]^{+}$} & 0.68 & 0.07 & -0.17 & 2.64 & 1.67 \\
$\mathrm{C}_{2} \mathrm{HF}$ & {$\left[\mathrm{C}_{8 \mathrm{mim}}\right]^{+}$} & 0.82 & 0.09 & -0.19 & 2.63 & 1.66 \\
$\mathrm{C}_{2} \mathrm{HF}$ & {$\left[\mathrm{P}_{4441}\right]^{+}$} & 1.22 & -0.02 & -0.16 & 2.92 & 1.44 \\
$\mathrm{C}_{2} \mathrm{HF}$ & {$\left[\mathrm{P}_{4448}\right]^{+}$} & 2.42 & -0.14 & -0.17 & 3.09 & 1.49 \\
$\mathrm{C}_{2} \mathrm{HF}$ & {$\left[\mathrm{P}_{8881}\right]^{+}$} & 2.56 & -0.06 & -0.15 & 2.98 & 1.51 \\
$\mathrm{C}_{2} \mathrm{HF}$ & {$\left[\mathrm{P}_{66614}\right]^{+}$} & 3.32 & -0.19 & -0.16 & 2.88 & 1.49 \\
$\mathrm{C}_{2} \mathrm{HF}$ & {$\left[\mathrm{P}_{88816}\right]^{+}$} & 4.04 & -0.18 & -0.16 & 2.90 & 1.50 \\
$\mathrm{C}_{4} \mathrm{HF}$ & {$\left[\mathrm{C}_{2} \mathrm{mim}\right]^{+}$} & 0.51 & -0.02 & -0.19 & 2.76 & 1.60 \\
$\mathrm{C}_{4} \mathrm{HF}$ & {$\left[\mathrm{C}_{8 \mathrm{mim}}\right]^{+}$} & 0.62 & -0.07 & -0.16 & 2.87 & 1.54 \\
$\mathrm{C}_{4} \mathrm{HF}$ & {$\left[\mathrm{P}_{4441}\right]^{+}$} & 0.93 & -0.09 & -0.18 & 3.03 & 1.45 \\
$\mathrm{C}_{4} \mathrm{HF}$ & {$\left[\mathrm{P}_{4448}\right]^{+}$} & 2.19 & -0.22 & -0.15 & 3.02 & 1.47 \\
$\mathrm{C}_{4} \mathrm{HF}$ & {$\left[\mathrm{P}_{8881}\right]^{+}$} & 2.23 & -0.06 & -0.15 & 2.83 & 1.53 \\
$\mathrm{C}_{4} \mathrm{HF}$ & {$\left[\mathrm{P}_{66614}\right]^{+}$} & 3.32 & -0.25 & -0.16 & 2.93 & 1.50 \\
$\mathrm{C}_{4} \mathrm{HF}$ & {$\left[\mathrm{P}_{88816}\right]^{+}$} & 4.08 & -0.24 & -0.15 & 2.95 & 1.40 \\
$\mathrm{C}_{8} \mathrm{HF}$ & {$\left[\mathrm{C}_{2} \mathrm{mim}\right]^{+}$} & 0.47 & 0.01 & -0.13 & 2.67 & 1.60 \\
$\mathrm{C}_{8} \mathrm{HF}$ & {$\left[\mathrm{C}_{8} \mathrm{mim}\right]^{+}$} & 0.78 & -0.06 & -0.15 & 2.93 & 1.51 \\
$\mathrm{C}_{8} \mathrm{HF}$ & {$\left[\mathrm{P}_{4441}\right]^{+}$} & 0.90 & -0.10 & -0.17 & 3.09 & 1.49 \\
$\mathrm{C}_{8} \mathrm{HF}$ & {$\left[\mathrm{P}_{4448}\right]^{+}$} & 2.34 & -0.25 & -0.17 & 3.19 & 1.41 \\
$\mathrm{C}_{8} \mathrm{HF}$ & {$\left[\mathrm{P}_{8881}\right]^{+}$} & 2.25 & -0.06 & -0.16 & 3.02 & 1.52 \\
$\mathrm{C}_{8} \mathrm{HF}$ & {$\left[\mathrm{P}_{66614}\right]^{+}$} & 2.48 & -0.15 & -0.16 & 3.06 & 1.49 \\
$\mathrm{C}_{8} \mathrm{HF}$ & {$\left[\mathrm{P}_{88816}\right]^{+}$} & 3.32 & -0.13 & -0.15 & 3.06 & 1.49 \\
\hline & & & & & &
\end{tabular}


Table S3. Pseudo equilibrium constants $K^{*}$ eq determined by the fluorescence intensity ratio.

\begin{tabular}{|c|c|c|c|c|c|c|c|}
\hline $\mathrm{C}_{\mathrm{n}} \mathrm{HF}$ & Cation & $450 \mathrm{~nm}$ & $430 \mathrm{~nm}$ & $410 \mathrm{~nm}$ & $390 \mathrm{~nm}$ & $370 \mathrm{~nm}$ & $360 \mathrm{~nm}$ \\
\hline $\mathrm{C}_{2} \mathrm{HF}$ & {$\left[\mathrm{C}_{2} \mathrm{mim}\right]^{+}$} & 0.20 & 0.30 & 0.45 & 0.55 & 0.33 & 0.43 \\
\hline $\mathrm{C}_{2} \mathrm{HF}$ & {$\left[\mathrm{C}_{8} \mathrm{mim}\right]^{+}$} & 0.24 & 0.36 & 0.55 & 0.49 & 0.45 & 0.55 \\
\hline $\mathrm{C}_{2} \mathrm{HF}$ & {$\left[\mathrm{P}_{4441}\right]^{+}$} & 0.32 & 0.54 & 0.53 & 0.85 & 0.79 & 0.71 \\
\hline $\mathrm{C}_{2} \mathrm{HF}$ & {$\left[\mathrm{P}_{4448}\right]^{+}$} & 0.54 & 0.83 & 1.12 & 1.51 & 1.38 & - \\
\hline $\mathrm{C}_{2} \mathrm{HF}$ & {$\left[\mathrm{P}_{8881}\right]^{+}$} & 0.55 & 0.86 & 1.26 & 1.54 & 1.55 & - \\
\hline $\mathrm{C}_{2} \mathrm{HF}$ & {$\left[\mathrm{P}_{66614}\right]^{+}$} & 0.56 & 1.17 & 1.52 & 1.92 & 2.01 & 2.00 \\
\hline $\mathrm{C}_{2} \mathrm{HF}$ & {$\left[\mathrm{P}_{88816}\right]^{+}$} & 0.79 & 1.38 & 1.92 & 2.24 & 2.48 & - \\
\hline $\mathrm{C}_{4} \mathrm{HF}$ & {$\left[\mathrm{C}_{2} \mathrm{mim}\right]^{+}$} & 0.21 & 0.21 & 0.34 & 0.26 & 0.26 & - \\
\hline $\mathrm{C}_{4} \mathrm{HF}$ & {$\left[\mathrm{C}_{8} \mathrm{mim}\right]^{+}$} & 0.25 & 0.25 & 0.54 & 0.55 & 0.48 & - \\
\hline $\mathrm{C}_{4} \mathrm{HF}$ & {$\left[\mathrm{P}_{4441}\right]^{+}$} & 0.31 & 0.28 & 0.58 & 0.80 & 0.68 & - \\
\hline $\mathrm{C}_{4} \mathrm{HF}$ & {$\left[\mathrm{P}_{4448}\right]^{+}$} & 0.50 & 0.75 & 0.99 & 1.28 & 1.26 & - \\
\hline $\mathrm{C}_{4} \mathrm{HF}$ & {$\left[\mathrm{P}_{8881}\right]^{+}$} & 0.50 & 0.77 & 1.09 & 1.35 & 1.36 & - \\
\hline $\mathrm{C}_{4} \mathrm{HF}$ & {$\left[\mathrm{P}_{66614}\right]^{+}$} & 0.52 & 1.15 & 1.47 & 1.90 & 1.91 & - \\
\hline $\mathrm{C}_{4} \mathrm{HF}$ & {$\left[\mathrm{P}_{88816}\right]^{+}$} & 0.74 & 1.35 & 1.89 & 2.20 & 2.33 & - \\
\hline $\mathrm{C}_{8} \mathrm{HF}$ & {$\left[\mathrm{C}_{2} \mathrm{mim}\right]^{+}$} & 0.18 & 0.10 & 0.27 & 0.38 & 0.29 & 0.21 \\
\hline $\mathrm{C}_{8} \mathrm{HF}$ & {$\left[\mathrm{C}_{8} \mathrm{mim}\right]^{+}$} & 0.22 & 0.29 & 0.30 & 0.56 & 0.40 & 0.56 \\
\hline $\mathrm{C}_{8} \mathrm{HF}$ & {$\left[\mathrm{P}_{4441}\right]^{+}$} & 0.26 & 0.21 & 0.30 & 0.65 & 0.46 & 0.68 \\
\hline $\mathrm{C}_{8} \mathrm{HF}$ & {$\left[\mathrm{P}_{4448}\right]^{+}$} & 0.50 & 0.85 & 1.06 & 1.36 & 1.26 & - \\
\hline $\mathrm{C}_{8} \mathrm{HF}$ & {$\left[\mathrm{P}_{8881}\right]^{+}$} & 0.49 & 0.79 & 1.13 & 1.36 & 1.31 & - \\
\hline $\mathrm{C}_{8} \mathrm{HF}$ & {$\left[\mathrm{P}_{66614}\right]^{+}$} & 0.46 & 0.93 & 1.22 & 1.53 & 1.49 & 1.71 \\
\hline $\mathrm{C}_{8} \mathrm{HF}$ & {$\left[\mathrm{P}_{88816}\right]^{+}$} & 0.68 & 1.22 & 1.75 & 1.89 & 2.11 & - \\
\hline
\end{tabular}


Table S4. Ionic concentration, several solvatochromic parameters, and viscosity $(\eta)$ of ILs used in this work.

\begin{tabular}{lcccccc}
\hline \multicolumn{1}{c}{ Cation } & $\begin{array}{c}\text { Ionic Conc. } \\
/ \mathrm{mol} \mathrm{dm}{ }^{-3}\end{array}$ & $\alpha^{R \text { a) }}$ & $\beta^{R \text { a) }}$ & $\pi_{\text {Band }}^{*}$ & $E_{T}^{N \text { a) }}$ & $\eta / \mathrm{cP}$ \\
\hline$\left[\mathrm{C}_{2} \mathrm{mim}\right]^{+}$ & $7.77^{\mathrm{b})}$ & 0.39 & 0.36 & $0.93^{\mathrm{b})}$ & 0.69 & $38^{\mathrm{c})}$ \\
{$\left[\mathrm{C}_{4} \mathrm{mim}\right]^{+}$} & $6.87^{\mathrm{b})}$ & 0.49 & 0.34 & $0.92^{\mathrm{b})}$ & 0.67 & $58^{\mathrm{d})}$ \\
{$\left[\mathrm{C}_{8 \mathrm{mim}}\right]^{+}$} & $5.57^{\mathrm{b})}$ & 0.39 & - & $0.91^{\mathrm{b})}$ & - & $90^{\mathrm{d})}$ \\
{$\left[\mathrm{P}_{4441}\right]^{+}$} & $5.15^{\mathrm{a})}$ & 0.41 & 0.31 & $0.91^{\mathrm{a})}$ & 0.50 & $207^{\mathrm{d})}$ \\
{$\left[\mathrm{P}_{4448}\right]^{+}$} & $3.96^{\mathrm{b})}$ & 0.34 & - & $0.90^{\mathrm{b})}$ & - & $250^{\mathrm{e})}$ \\
{$\left[\mathrm{P}_{8881}\right]^{+}$} & $3.33^{\mathrm{b})}$ & 0.34 & - & $0.88^{\mathrm{b})}$ & - & $423^{\mathrm{f}}$ \\
{$\left[\mathrm{P}_{66614}\right]^{+}$} & $2.80^{\mathrm{a})}$ & 0.36 & 0.37 & $0.84^{\mathrm{a})}$ & 0.45 & $460^{\mathrm{c})}$ \\
{$\left[\mathrm{P}_{88816}\right]^{+}$} & $2.35^{\mathrm{b})}$ & 0.27 & - & $0.82^{\mathrm{b})}$ & - & $417^{\mathrm{f}}$ \\
acetonitrile & 0 & 0.30 & 0.46 & $0.80^{\mathrm{a})}$ & 0.46 & $0.34^{\mathrm{g})}$ \\
\hline
\end{tabular}

a) ref. 8. b) ref. 6. c) ref. 2. d) ref. 3. e) ref. 5. f) this work $\left(22^{\circ} \mathrm{C}\right)$. g) ref. 9 . 
Table S5(a). Parameters obtained from the fit to Eq. (6) of the time profile of the band integral of IE of $\mathrm{T}^{*}$ excited at $400 \mathrm{~nm}$.

\begin{tabular}{cccccccc}
\hline $\mathrm{C}_{\mathrm{n}} \mathrm{HF}$ & $\mathrm{Cation}_{1}$ & $T_{1}$ & $\tau_{1} / \mathrm{ps}$ & $T_{2}$ & $\tau_{2} / \mathrm{ps}$ & $\tau_{\text {ESIPT }} / \mathrm{ps}$ & $\tau_{3} / \mathrm{ns}$ \\
\hline $\mathrm{C}_{2} \mathrm{HF}$ & {$\left[\mathrm{C}_{2} \mathrm{mim}\right]^{+}$} & 0.42 & 4.1 & 0.59 & 25.9 & 16.7 & $2.3^{\mathrm{a})}$ \\
$\mathrm{C}_{2} \mathrm{HF}$ & {$\left[\mathrm{C}_{8} \mathrm{mim}\right]^{+}$} & 0.46 & 3.6 & 0.54 & 22.3 & 13.7 & $2.7^{\mathrm{b})}$ \\
$\mathrm{C}_{2} \mathrm{HF}$ & {$\left[\mathrm{P}_{4441}\right]^{+}$} & 0.44 & 3.6 & 0.56 & 16.8 & 11.0 & $2.8^{\mathrm{a})}$ \\
$\mathrm{C}_{2} \mathrm{HF}$ & {$\left[\mathrm{P}_{4448}\right]^{+}$} & 0.44 & 3.6 & 0.56 & 18.8 & 12.1 & $2.3^{\mathrm{c})}$ \\
$\mathrm{C}_{2} \mathrm{HF}$ & {$\left[\mathrm{P}_{8881}\right]^{+}$} & 0.50 & 3.5 & 0.50 & 16.8 & 10.1 & $2.5^{\mathrm{c}}$ \\
$\mathrm{C}_{2} \mathrm{HF}$ & {$\left[\mathrm{P}_{66614}\right]^{+}$} & 0.58 & 3.6 & 0.42 & 23.5 & 11.9 & $2.5^{\mathrm{a})}$ \\
$\mathrm{C}_{2} \mathrm{HF}$ & {$\left[\mathrm{P}_{88816}\right]^{+}$} & 0.43 & 3.8 & 0.57 & 13.1 & 9.1 & $2.5^{\mathrm{c})}$ \\
$\mathrm{C}_{4} \mathrm{HF}$ & {$\left[\mathrm{C}_{2} \mathrm{mim}\right]^{+}$} & 0.38 & 4.4 & 0.62 & 23.8 & 16.4 & $2.3^{\mathrm{c})}$ \\
$\mathrm{C}_{4} \mathrm{HF}$ & {$\left[\mathrm{C}_{8} \mathrm{mim}\right]^{+}$} & 0.34 & 3.1 & 0.66 & 18.9 & 13.5 & $2.7^{\mathrm{c})}$ \\
$\mathrm{C}_{4} \mathrm{HF}$ & {$\left[\mathrm{P}_{4441}\right]^{+}$} & 0.39 & 3.2 & 0.61 & 16.4 & 11.3 & $2.8^{\mathrm{c})}$ \\
$\mathrm{C}_{4} \mathrm{HF}$ & {$\left[\mathrm{P}_{4448}\right]^{+}$} & 0.46 & 3.5 & 0.54 & 19.3 & 12.0 & $2.3^{\mathrm{c})}$ \\
$\mathrm{C}_{4} \mathrm{HF}$ & {$\left[\mathrm{P}_{8881}\right]^{+}$} & 0.47 & 4.0 & 0.53 & 20.4 & 12.7 & $2.5^{\mathrm{c})}$ \\
$\mathrm{C}_{4} \mathrm{HF}$ & {$\left[\mathrm{P}_{66614}\right]^{+}$} & 0.55 & 4.0 & 0.45 & 19.9 & 11.1 & $2.5^{\mathrm{c})}$ \\
$\mathrm{C}_{4} \mathrm{HF}$ & {$\left[\mathrm{P}_{88816}\right]^{+}$} & 0.58 & 4.1 & 0.42 & 18.9 & 10.3 & $2.5^{\mathrm{c})}$ \\
\hline $\mathrm{C}_{8} \mathrm{HF}$ & {$\left[\mathrm{C}_{2} \mathrm{mim}\right]^{+}$} & 0.35 & 5.8 & 0.65 & 22.6 & 16.7 & $2.3^{\mathrm{c})}$ \\
$\mathrm{C}_{8} \mathrm{HF}$ & {$\left[\mathrm{C}_{8} \mathrm{mim}\right]^{+}$} & 0.37 & 2.7 & 0.63 & 20.0 & 13.6 & $2.7^{\mathrm{d})}$ \\
$\mathrm{C}_{8} \mathrm{HF}$ & {$\left[\mathrm{P}_{4441}\right]^{+}$} & 0.37 & 4.2 & 0.63 & 20.6 & 14.5 & $2.8^{\mathrm{d})}$ \\
$\mathrm{C}_{8} \mathrm{HF}$ & {$\left[\mathrm{P}_{4448}\right]^{+}$} & 0.46 & 3.1 & 0.54 & 20.3 & 12.4 & $2.3^{\mathrm{d})}$ \\
$\mathrm{C}_{8} \mathrm{HF}$ & {$\left[\mathrm{P}_{8881}\right]^{+}$} & 0.38 & 2.7 & 0.62 & 19.4 & 13.0 & $2.5^{\mathrm{d})}$ \\
$\mathrm{C}_{8} \mathrm{HF}$ & {$\left[\mathrm{P}_{66614}\right]^{+}$} & 0.48 & 2.7 & 0.52 & 19.3 & 11.3 & $2.5^{\mathrm{c})}$ \\
$\mathrm{C}_{8} \mathrm{HF}$ & {$\left[\mathrm{P}_{88816}\right]^{+}$} & 0.49 & 3.5 & 0.51 & 16.5 & 10.1 & $2.5^{\mathrm{d})}$ \\
\hline
\end{tabular}
a) Taken from ref. 3.
b) In the ref. 3, ESIPT time constant of DEAHF in [Omim] $\left[\mathrm{NTf}_{2}\right]$ was mistakenly calculated as $10.9 \pm 2.4$ ps. Actually, $14.6 \mathrm{ps}$ is correct.
c) This work. The value was determined from the fluorescence measurement excited at $410 \mathrm{~nm}$.
d)The value is assumed to be the same as the one of $\mathrm{C}_{2} \mathrm{HF}$ in the same solvent. 
Table S5(b). Parameters obtained from the fit to Eq. (6) of the time profile of $\Delta \mathrm{OD}$ at the band center of IE of $\mathrm{T}^{*}$ excited at $400 \mathrm{~nm}$.

\begin{tabular}{cccccccc}
\hline $\mathrm{C}_{\mathrm{n}} \mathrm{HF}$ & $\mathrm{Cation}_{2}$ & $T_{1}$ & $\tau_{1} / \mathrm{ps}$ & $T_{2}$ & $\tau_{2} / \mathrm{ps}$ & $\tau_{\text {ESIPT }} / \mathrm{ps}$ & $\tau_{3} / \mathrm{ns}{ }^{\mathrm{a})}$ \\
\hline $\mathrm{C}_{2} \mathrm{HF}$ & {$\left[\mathrm{C}_{2} \mathrm{mim}\right]^{+}$} & 0.47 & 2.3 & 0.53 & 32.5 & 18.3 & 2.3 \\
$\mathrm{C}_{2} \mathrm{HF}$ & {$\left[\mathrm{C}_{8} \mathrm{mim}\right]^{+}$} & 0.33 & 2.9 & 0.67 & 22.1 & 15.8 & 2.7 \\
$\mathrm{C}_{2} \mathrm{HF}$ & {$\left[\mathrm{P}_{4441}\right]^{+}$} & 0.38 & 2.1 & 0.62 & 16.5 & 11.0 & 2.8 \\
$\mathrm{C}_{2} \mathrm{HF}$ & {$\left[\mathrm{P}_{4448}\right]^{+}$} & 0.32 & 2.3 & 0.68 & 17.0 & 12.3 & 2.3 \\
$\mathrm{C}_{2} \mathrm{HF}$ & {$\left[\mathrm{P}_{8881}\right]^{+}$} & 0.34 & 2.0 & 0.66 & 17.0 & 11.9 & 2.5 \\
$\mathrm{C}_{2} \mathrm{HF}$ & {$\left[\mathrm{P}_{66614}\right]^{+}$} & 0.45 & 3.8 & 0.55 & 17.7 & 11.4 & 2.5 \\
$\mathrm{C}_{2} \mathrm{HF}$ & {$\left[\mathrm{P}_{88816}\right]^{+}$} & 0.47 & 2.0 & 0.53 & 15.4 & 9.1 & 2.5 \\
$\mathrm{C}_{4} \mathrm{HF}$ & {$\left[\mathrm{C}_{2} \mathrm{mim}\right]^{+}$} & 0.40 & 2.6 & 0.60 & 25.3 & 16.2 & 2.3 \\
$\mathrm{C}_{4} \mathrm{HF}$ & {$\left[\mathrm{C}_{8} \mathrm{mim}\right]^{+}$} & 0.33 & 2.1 & 0.67 & 23.6 & 16.5 & 2.7 \\
$\mathrm{C}_{4} \mathrm{HF}$ & {$\left[\mathrm{P}_{4441}\right]^{+}$} & 0.38 & 2.3 & 0.62 & 16.8 & 11.3 & 2.8 \\
$\mathrm{C}_{4} \mathrm{HF}$ & {$\left[\mathrm{P}_{4448}\right]^{+}$} & 0.40 & 2.5 & 0.60 & 17.3 & 11.4 & 2.3 \\
$\mathrm{C}_{4} \mathrm{HF}$ & {$\left[\mathrm{P}_{8881}\right]^{+}$} & 0.41 & 2.5 & 0.59 & 18.0 & 11.6 & 2.5 \\
$\mathrm{C}_{4} \mathrm{HF}$ & {$\left[\mathrm{P}_{66614}\right]^{+}$} & 0.42 & 2.3 & 0.58 & 16.7 & 10.7 & 2.5 \\
$\mathrm{C}_{4} \mathrm{HF}$ & {$\left[\mathrm{P}_{88816}\right]^{+}$} & 0.58 & 4.4 & 0.42 & 19.7 & 10.8 & 2.5 \\
$\mathrm{C}_{8} \mathrm{HF}$ & {$\left[\mathrm{C}_{2} \mathrm{mim}\right]^{+}$} & 0.37 & 2.4 & 0.63 & 29.0 & 19.2 & 2.3 \\
$\mathrm{C}_{8} \mathrm{HF}$ & {$\left[\mathrm{C}_{8} \text { mim }\right]^{+}$} & 0.30 & 2.1 & 0.70 & 24.6 & 17.8 & 2.7 \\
$\mathrm{C}_{8} \mathrm{HF}$ & {$\left[\mathrm{P}_{4441}\right]^{+}$} & 0.43 & 3.6 & 0.57 & 21.0 & 13.5 & 2.8 \\
$\mathrm{C}_{8} \mathrm{HF}$ & {$\left[\mathrm{P}_{4448}\right]^{+}$} & 0.43 & 2.7 & 0.57 & 20.5 & 12.9 & 2.3 \\
$\mathrm{C}_{8} \mathrm{HF}$ & {$\left[\mathrm{P}_{8881}\right]^{+}$} & 0.48 & 2.7 & 0.52 & 22.7 & 13.1 & 2.5 \\
$\mathrm{C}_{8} \mathrm{HF}$ & {$\left[\mathrm{P}_{66614}\right]^{+}$} & 0.42 & 2.3 & 0.58 & 19.9 & 12.5 & 2.5 \\
$\mathrm{C}_{8} \mathrm{HF}$ & {$\left[\mathrm{P}_{88816}\right]^{+}$} & 0.35 & 2.2 & 0.65 & 16.2 & 11.3 & 2.5 \\
\hline
\end{tabular}

a) The values are the same as those in Table S5(a). 
Table S6(a). Parameters obtained from the fit to Eq. (6) of the time profile of $\Delta \mathrm{OD}$ at the band center of IE of $\mathrm{T}^{*}$ excited at $360 \mathrm{~nm}$.

\begin{tabular}{cccccccc}
\hline $\mathrm{C}_{\mathrm{n}} \mathrm{HF}$ & Cation & $T_{1}$ & $\tau_{1} / \mathrm{ps}$ & $T_{2}$ & $\tau_{2} / \mathrm{ps}$ & $\tau_{\text {ESIPT }} / \mathrm{ps}$ & $\left.\tau_{3} / \mathrm{ns}{ }^{\mathrm{a}}\right)$ \\
\hline $\mathrm{C}_{2} \mathrm{HF}$ & {$\left[\mathrm{C}_{2} \mathrm{mim}\right]^{+}$} & 0.59 & 4.4 & 0.41 & 35.0 & 16.9 & 2.3 \\
$\mathrm{C}_{2} \mathrm{HF}$ & {$\left[\mathrm{C}_{8} \mathrm{mim}\right]^{+}$} & 0.62 & 3.2 & 0.38 & 34.4 & 15.0 & 2.7 \\
$\mathrm{C}_{2} \mathrm{HF}$ & {$\left[\mathrm{P}_{4441}\right]^{+}$} & 0.33 & 2.2 & 0.67 & 20.8 & 14.7 & 2.8 \\
$\mathrm{C}_{2} \mathrm{HF}$ & {$\left[\mathrm{P}_{66614}\right]^{+}$} & 0.47 & 3.5 & 0.53 & 18.6 & 11.5 & 2.5 \\
\hdashline $\mathrm{C}_{8} \mathrm{HF}$ & {$\left[\mathrm{C}_{2} \mathrm{mim}\right]^{+}$} & 0.58 & 4.9 & 0.42 & 28.1 & 14.6 & 2.3 \\
$\mathrm{C}_{8} \mathrm{HF}$ & {$\left[\mathrm{C}_{8} \mathrm{mim}\right]^{+}$} & 0.53 & 3.6 & 0.47 & 31.6 & 16.8 & 2.7 \\
$\mathrm{C}_{8} \mathrm{HF}$ & {$\left[\mathrm{P}_{4441}\right]^{+}$} & 0.45 & 3.0 & 0.55 & 25.6 & 15.4 & 2.8 \\
$\mathrm{C}_{8} \mathrm{HF}$ & {$\left[\mathrm{P}_{66614}\right]^{+}$} & 0.35 & 2.4 & 0.65 & 20.3 & 14.0 & 2.5 \\
\hline
\end{tabular}

a)The value is the same as the one in corresponding solute and solvent in Table S5(a) assuming that the fluorescence lifetime is not dependent on the excitation wavelength.

Table S6(b). Parameters obtained from the fit to Eq. (6) of the time profile of $\Delta \mathrm{OD}$ at the band center of IE of $\mathrm{T}^{*}$ excited at $450 \mathrm{~nm}$.

\begin{tabular}{cccccccc}
\hline $\mathrm{C}_{\mathrm{n}} \mathrm{HF}$ & Cation & $T_{1}$ & $\tau_{1} / \mathrm{ps}$ & $T_{2}$ & $\tau_{2} / \mathrm{ps}$ & $\tau_{\text {ESIPT }} / \mathrm{ps}$ & $\tau_{3} / \mathrm{ns}$ \\
\hline $\mathrm{C}_{2} \mathrm{HF}$ & {$\left[\mathrm{C}_{2} \mathrm{mim}\right]^{+}$} & 0.38 & 4.8 & 0.62 & 54.1 & 35.4 & 2.3 \\
$\mathrm{C}_{2} \mathrm{HF}$ & {$\left[\mathrm{C}_{8} \mathrm{mim}\right]^{+}$} & 0.44 & 4.3 & 0.56 & 41.8 & 25.3 & 2.7 \\
$\mathrm{C}_{2} \mathrm{HF}$ & {$\left[\mathrm{P}_{4441}\right]^{+}$} & 0.61 & 3.6 & 0.39 & 49.7 & 21.6 & 2.8 \\
$\mathrm{C}_{2} \mathrm{HF}$ & {$\left[\mathrm{P}_{66614}\right]^{+}$} & 0.55 & 6.0 & 0.45 & 40.8 & 21.6 & 2.5 \\
$\mathrm{C}_{8} \mathrm{HF}$ & {$\left[\mathrm{C}_{2} \mathrm{mim}\right]^{+}$} & 0.31 & 2.4 & 0.69 & 44.0 & 31.1 & 2.3 \\
$\mathrm{C}_{8} \mathrm{HF}$ & {$\left[\mathrm{C}_{8} \mathrm{mim}\right]^{+}$} & 0.44 & 4.8 & 0.56 & 46.1 & 27.9 & 2.7 \\
$\mathrm{C}_{8} \mathrm{HF}$ & {$\left[\mathrm{P}_{4441}\right]^{+}$} & 0.59 & 5.3 & 0.41 & 45.0 & 21.6 & 2.8 \\
$\mathrm{C}_{8} \mathrm{HF}$ & {$\left[\mathrm{P}_{66614}\right]^{+}$} & 0.57 & 6.3 & 0.43 & 55.8 & 27.6 & 2.5 \\
\hline
\end{tabular}

a)The value is the same as the one in corresponding solute and solvent in Table S5(a) assuming that the fluorescence lifetime is not dependent on the excitation wavelength. 
Table S7(a). Parameters obtained from the fit to Eq. (8) of the time profile of the apparent negative peak position of the GSB excited at $450 \mathrm{~nm}$.

\begin{tabular}{cccccccc}
\hline $\mathrm{C}_{\mathrm{n}} \mathrm{HF}$ & Cation & $v_{\infty}$ & $\begin{array}{c}\Delta v_{1} / \\
10^{3} \mathrm{~cm}^{-1}\end{array}$ & $\tau_{1} / \mathrm{ps}$ & $\begin{array}{c}\Delta v_{2} / \\
10^{3} \mathrm{~cm}^{-1}\end{array}$ & $\tau_{2} / \mathrm{ps}$ & $\tau_{\text {ave }} / \mathrm{ps}$ \\
\hline $\mathrm{C}_{2} \mathrm{HF}$ & {$\left[\mathrm{C}_{2} \mathrm{mim}\right]^{+}$} & 25.0 & -0.45 & 7.6 & -0.72 & 107 & 68.9 \\
$\mathrm{C}_{2} \mathrm{HF}$ & {$\left[\mathrm{C}_{8} \mathrm{mim}\right]^{+}$} & 24.7 & -0.29 & 5.4 & -0.85 & 134 & 101 \\
$\mathrm{C}_{2} \mathrm{HF}$ & {$\left[\mathrm{P}_{4441}\right]^{+}$} & 24.6 & -0.41 & 5.6 & -0.79 & 108 & 72.8 \\
$\mathrm{C}_{2} \mathrm{HF}$ & {$\left[\mathrm{P}_{66614}\right]^{+}$} & 24.1 & -0.33 & 4.5 & -0.40 & 121 & 68.1 \\
$\mathrm{C}_{8} \mathrm{HF}$ & {$\left[\mathrm{C}_{2} \mathrm{mim}\right]^{+}$} & 24.8 & -0.39 & 6.1 & -0.66 & 115 & 74.7 \\
$\mathrm{C}_{8} \mathrm{HF}$ & {$\left[\mathrm{C}_{8} \mathrm{mim}\right]^{+}$} & 24.1 & -0.44 & 4.1 & -1.05 & 81 & 58.2 \\
$\mathrm{C}_{8} \mathrm{HF}$ & {$\left[\mathrm{P}_{4441}\right]^{+}$} & 24.1 & -0.52 & 3.3 & -0.52 & 110 & 56.6 \\
$\mathrm{C}_{8} \mathrm{HF}$ & {$\left[\mathrm{P}_{66614}\right]^{+}$} & 24.2 & -0.44 & 6.5 & -0.45 & 143.0 & 75.5 \\
\hline
\end{tabular}

Table S7(b). Parameters obtained from the fit to Eq. (8) of the apparent peak position of IE of $\mathrm{N}^{*}$ of $\mathrm{C}_{2} \mathrm{HF}$ and the fluorescence peak position of $\mathrm{C}_{2} \mathrm{HF}$ excited at $450 \mathrm{~nm}$.

\begin{tabular}{cccccccc}
\hline Data & Cation & $v_{\infty}$ & $\begin{array}{c}\Delta v_{1} / \\
10^{3} \mathrm{~cm}^{-1}\end{array}$ & $\tau_{1} / \mathrm{ps}$ & $\begin{array}{c}\Delta v_{2} / \\
10^{3} \mathrm{~cm}^{-1}\end{array}$ & $\tau_{2} / \mathrm{ps}$ & $\tau_{\text {ave }} / \mathrm{ps}$ \\
\hline IE & {$\left[\mathrm{C}_{2} \mathrm{mim}\right]^{+}$} & 18.7 & 0.52 & 0.6 & 1.61 & 33.9 & 25.8 \\
IE & {$\left[\mathrm{C}_{8} \mathrm{mim}\right]^{+}$} & 19.7 & 0.47 & 0.7 & 0.79 & 35.9 & 22.8 \\
IE & {$\left[\mathrm{P}_{4441}\right]^{+}$} & 19.8 & 0.23 & 1.0 & 0.73 & 83.3 & 63.6 \\
IE & {$\left[\mathrm{P}_{66614}\right]^{+}$} & 20.4 & 0.45 & 1.3 & 0.64 & 47.6 & 28.5 \\
Fluorescence $^{\text {a) }}$ & {$\left[\mathrm{C}_{2} \mathrm{mim}\right]^{+}$} & 18.9 & 0.79 & 1.2 & 1.23 & 41.2 & 25.6 \\
Fluorescence $^{\text {a) }}$ & {$\left[\mathrm{C}_{8} \mathrm{mim}\right]^{+}$} & 19.7 & 0.49 & 0.7 & 0.7 & 45.3 & 27.0 \\
Fluorescence $^{\text {a) }}$ & {$\left[\mathrm{P}_{4441}\right]^{+}$} & 19.6 & 0.46 & 0.8 & 0.98 & 47.0 & 32.2 \\
Fluorescence $^{\mathrm{a} \text { b) }}$ & {$\left[\mathrm{P}_{66614}\right]^{+}$} & 20.4 & 0.26 & $1.3^{\mathrm{c})}$ & 0.82 & 36.5 & 28.0 \\
\hline
\end{tabular}

a) Data taken from Ref. 3.

b) Data obtained by excitation at $430 \mathrm{~nm}$.

c) The value of $\tau_{1}$ was fixed to the value obtained from the fit of the IE. 
Figure S1. Spectrum of the pump pulse used for transient absorption measurement. Dashed line represents the equilibrium absorption spectrum of $\mathrm{C}_{2} \mathrm{HF}$ in $\left[\mathrm{P}_{4441}\right]\left[\mathrm{NTf}_{2}\right]$.

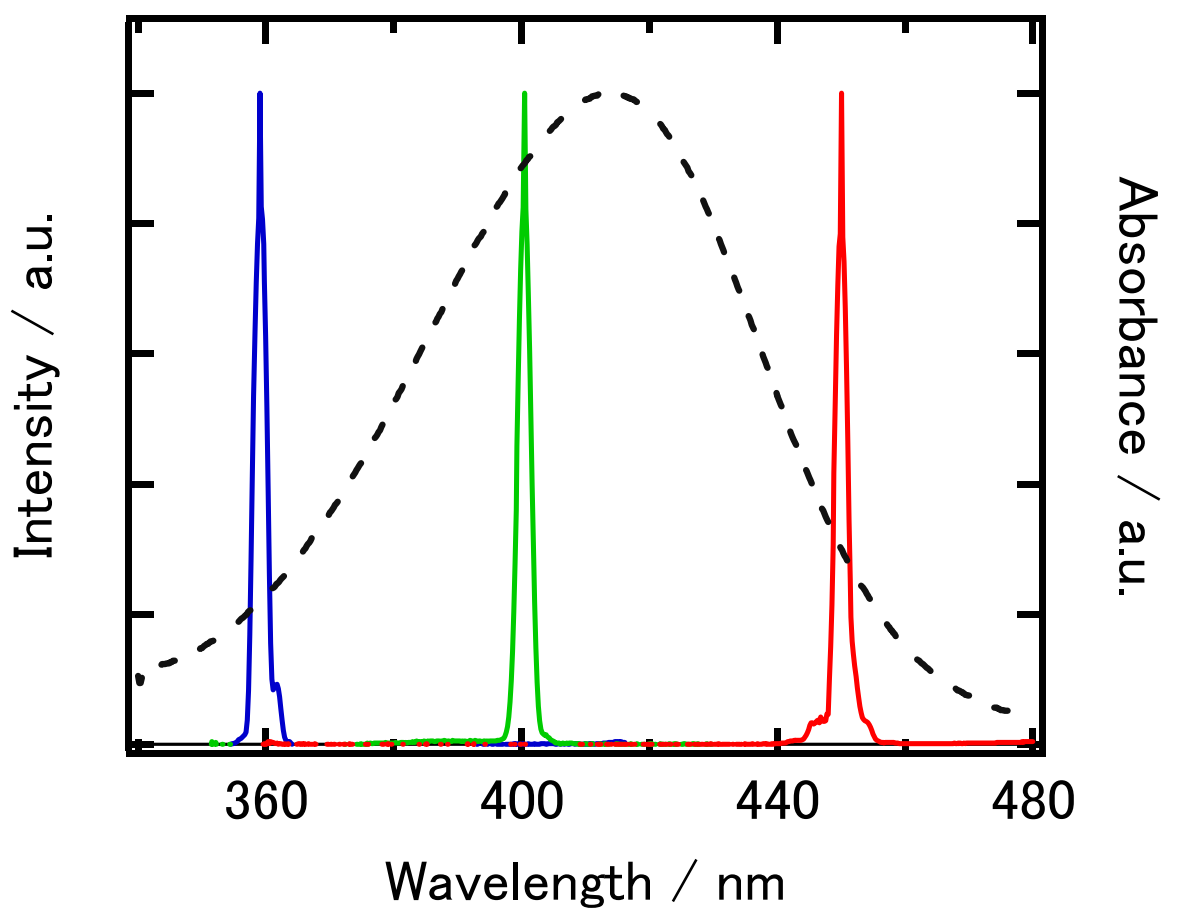


Figure S2. Absorption spectrum (blue) and fluorescence spectrum (solid green) excited at $400 \mathrm{~nm}$ of (17) $\mathrm{C}_{2} \mathrm{HF}$, (8-14) $\mathrm{C}_{4} \mathrm{HF}$ and (15-21) $\mathrm{C}_{8} \mathrm{HF}$. Dashed green lines represent the decomposed spectra into $\mathrm{N}^{*}$ and $\mathrm{T}^{*}$ species. Indices in the figures represent the carbon numbers of $\mathrm{C}_{\mathrm{n}} \mathrm{HF}$ and ILs.

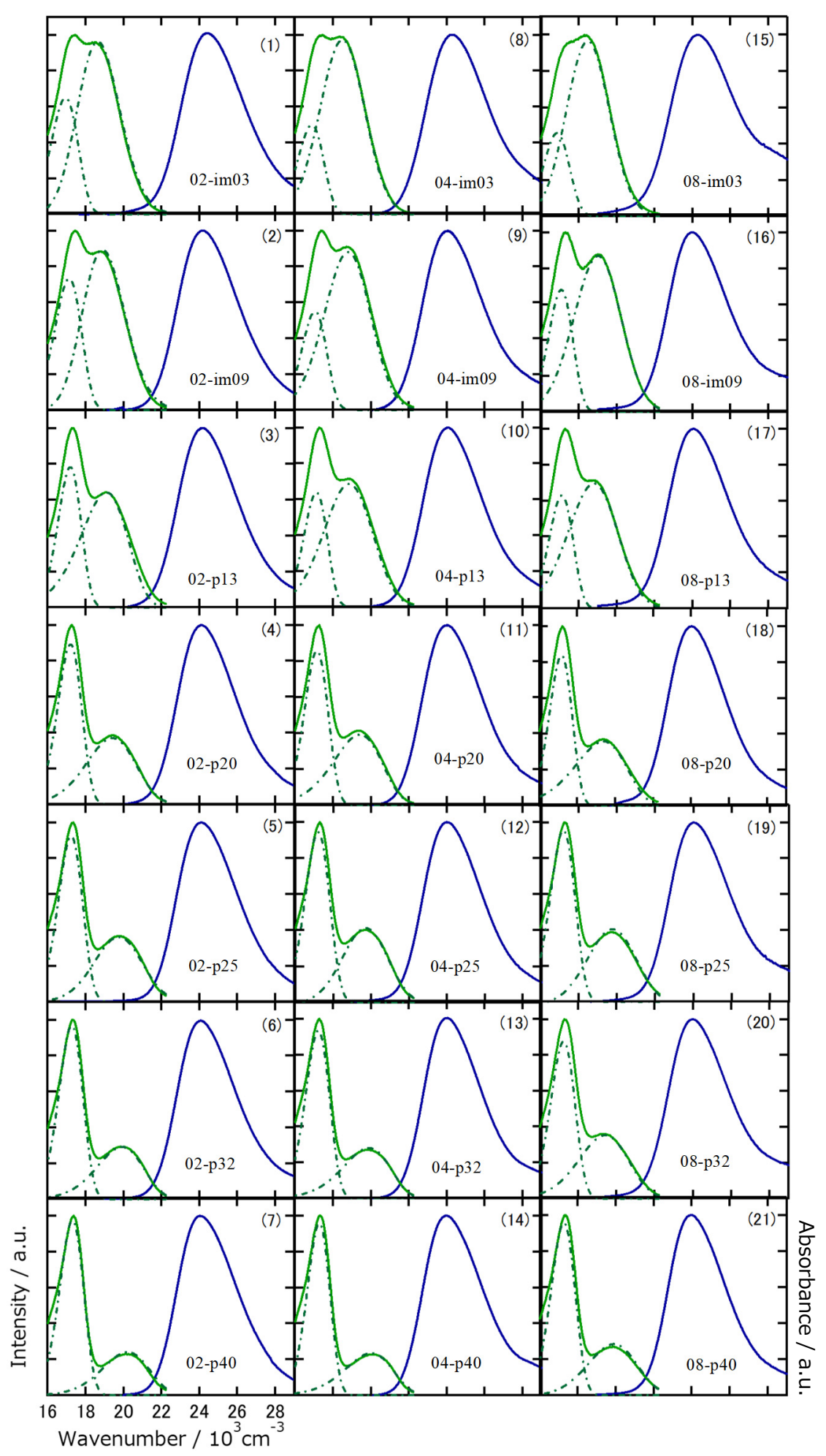


Figure S3. Plot of the absorbance band center against the carbon number of the alkyl chain of the cation of the IL. The open markers represent those in the imidazolium-cation based ILs, and the filled ones those in the phosphonium-cation based ILs.

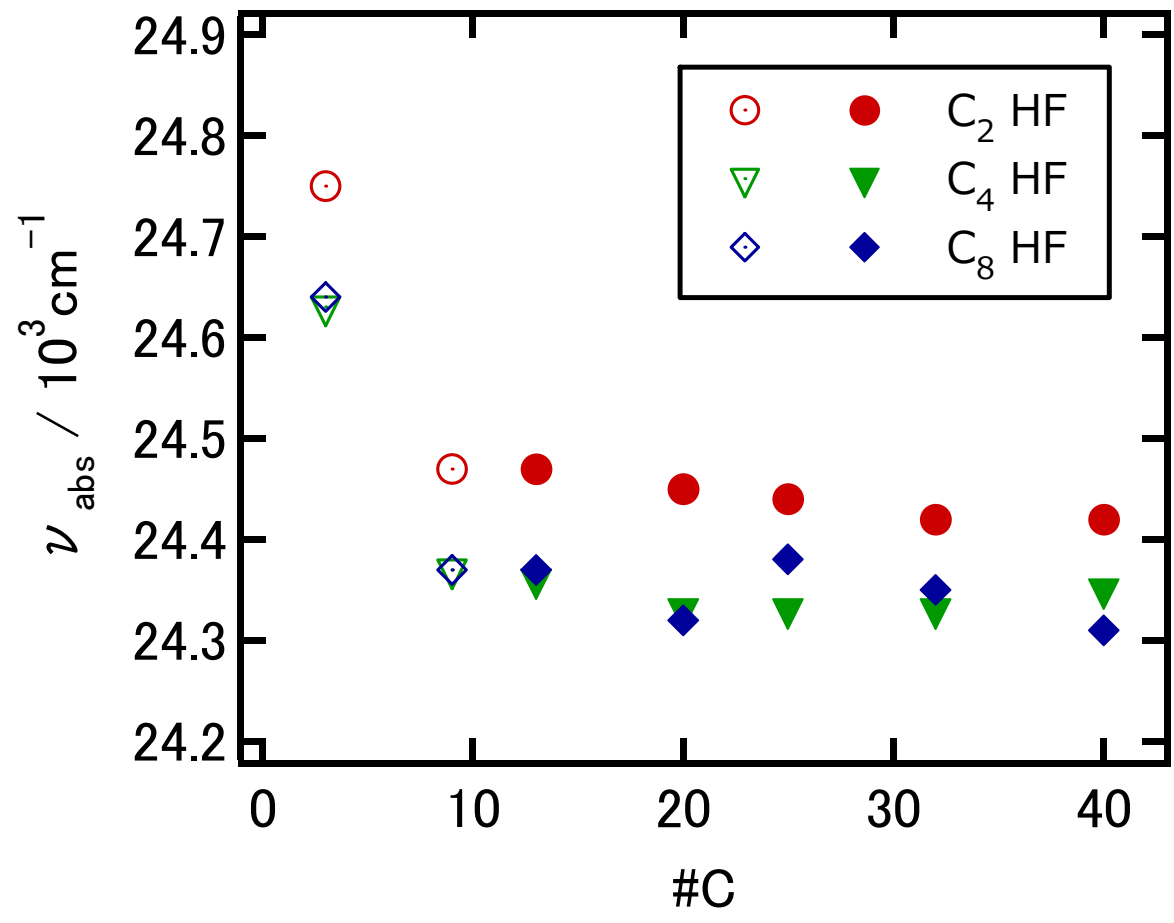


Figure S4. 3D plots (a) and (b) of the transient absorption spectra of $\mathrm{C}_{2} \mathrm{HF}$ in (a) $\left[\mathrm{C}_{2}\right.$ mim $]\left[\mathrm{NTf}_{2}\right]$ and (b) $\left[\mathrm{P}_{66614}\right]\left[\mathrm{NTf}_{2}\right]$. In graphs, red represents positive, and blue negative. The color chart of the 3D plot is shown as the side bar in the plot.

(a)

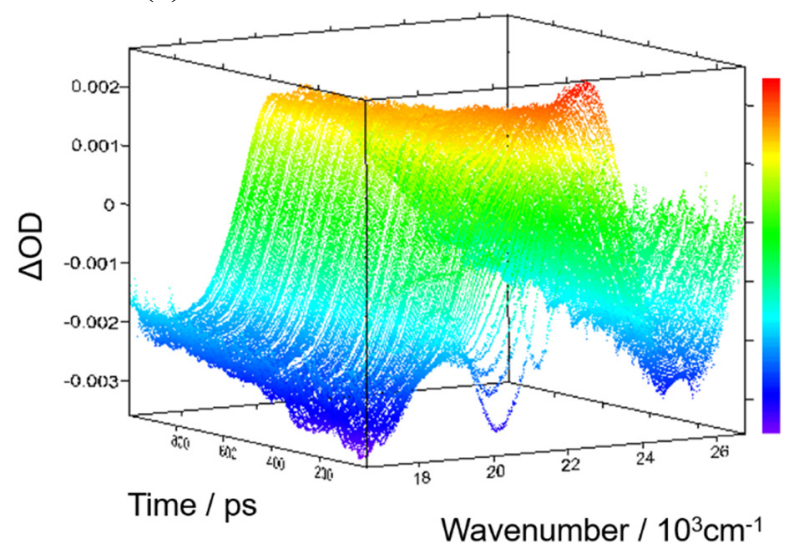

(b)

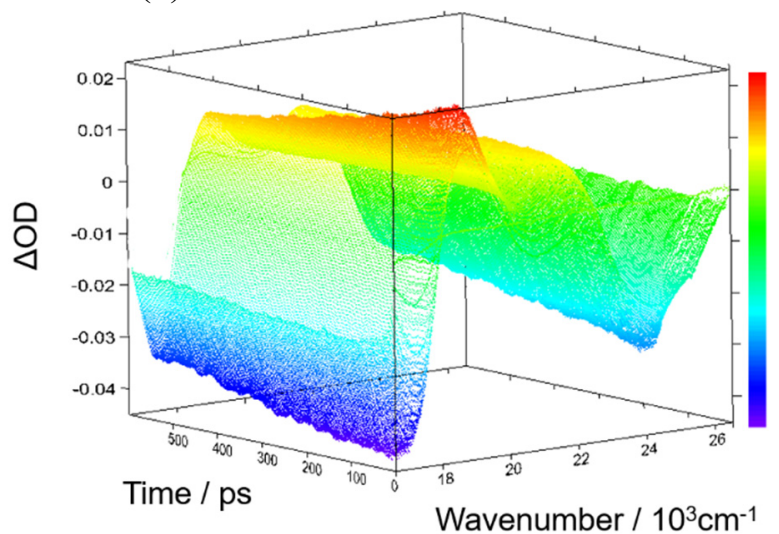


Figure S5. Transient absorption spectra of $\mathrm{C}_{2} \mathrm{HF}$ in $\left[\mathrm{C}_{2} \mathrm{mim}\right]\left[\mathrm{NTf}_{2}\right](\mathrm{a}, \mathrm{b})$ and $\left[\mathrm{P}_{4441}\right]\left[\mathrm{NTf}_{2}\right](\mathrm{c}, \mathrm{d})$ after excitation at $360 \mathrm{~nm}(\mathrm{a}, \mathrm{c})$ and $450 \mathrm{~nm}(\mathrm{~b}, \mathrm{~d})$.

(a)

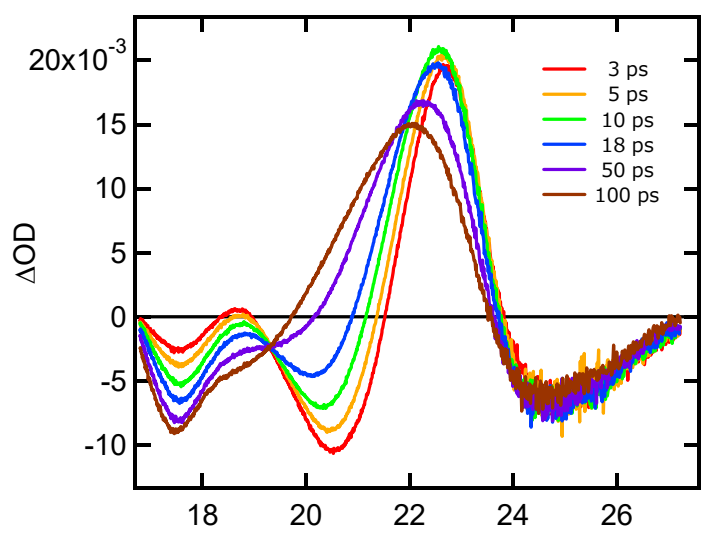

(c)

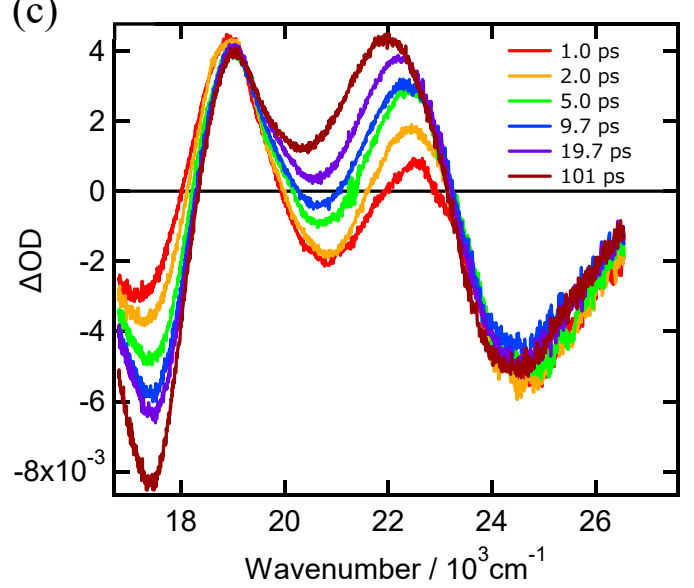

(b)

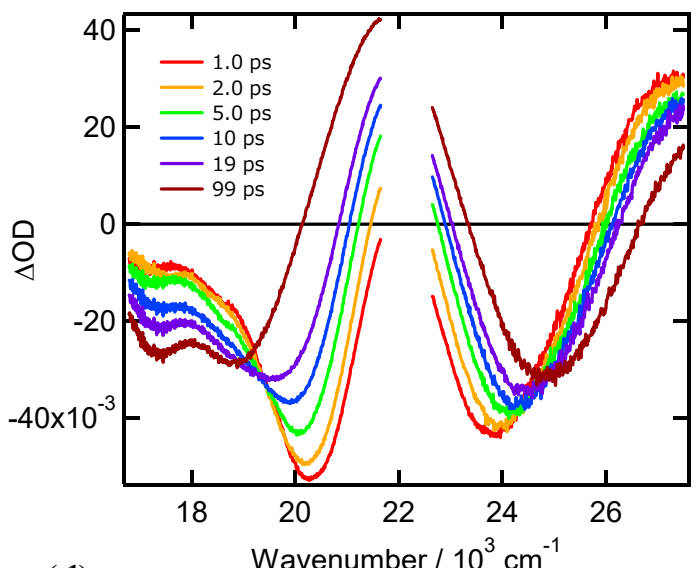

(d)

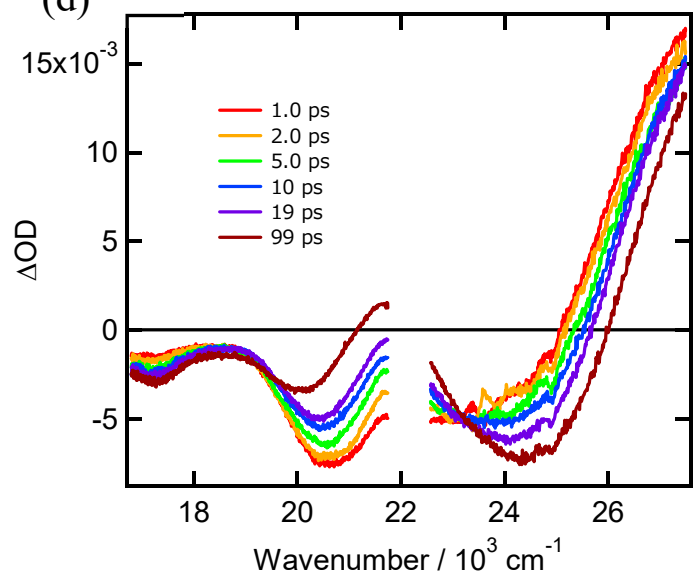


Figure S6. Comparison of time profiles between the band integral and $\triangle \mathrm{OD}$ of $\mathrm{C}_{2} \mathrm{HF}$ in $\left[\mathrm{P}_{4441}\right]\left[\mathrm{NTf}_{2}\right]$. The red circles are the results from the band integral of the $T^{*} \mathrm{IE}$ obtained by the spectral decomposition and blue + markers are those from $\triangle \mathrm{OD}$ at the band center of the $\mathrm{T}^{*} \mathrm{IE}$. The inset shows the figure with logarithmic horizontal scale.

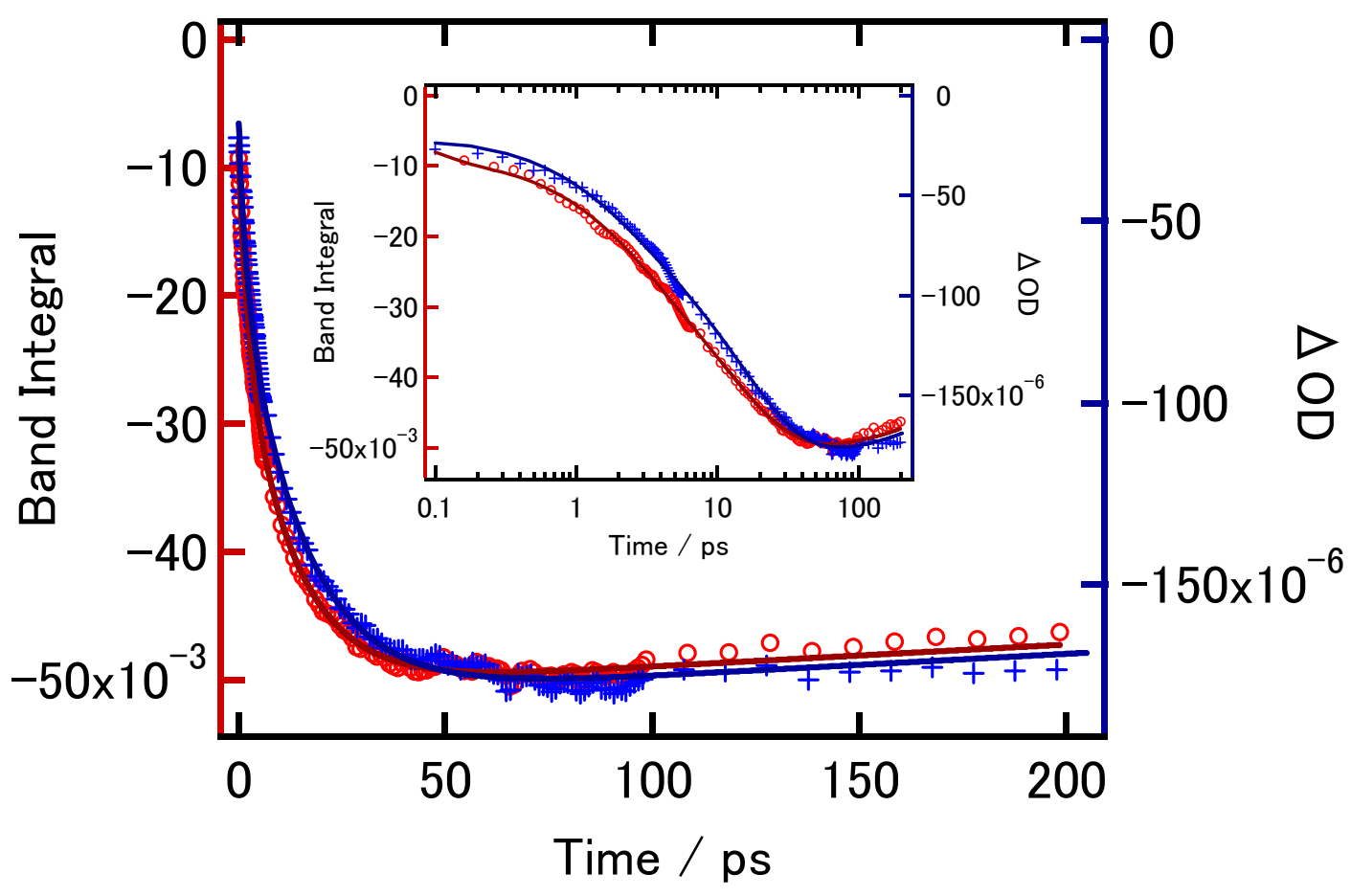


Figure S7(a-1). Time profile of the band integral of IE of $\mathrm{T}^{*}$ for $\mathrm{C}_{2} \mathrm{HF}$ excited at $400 \mathrm{~nm}$. The band integral data are evaluated from the spectral deconvolution analysis. Black lines represent the fitting to Eq (6).
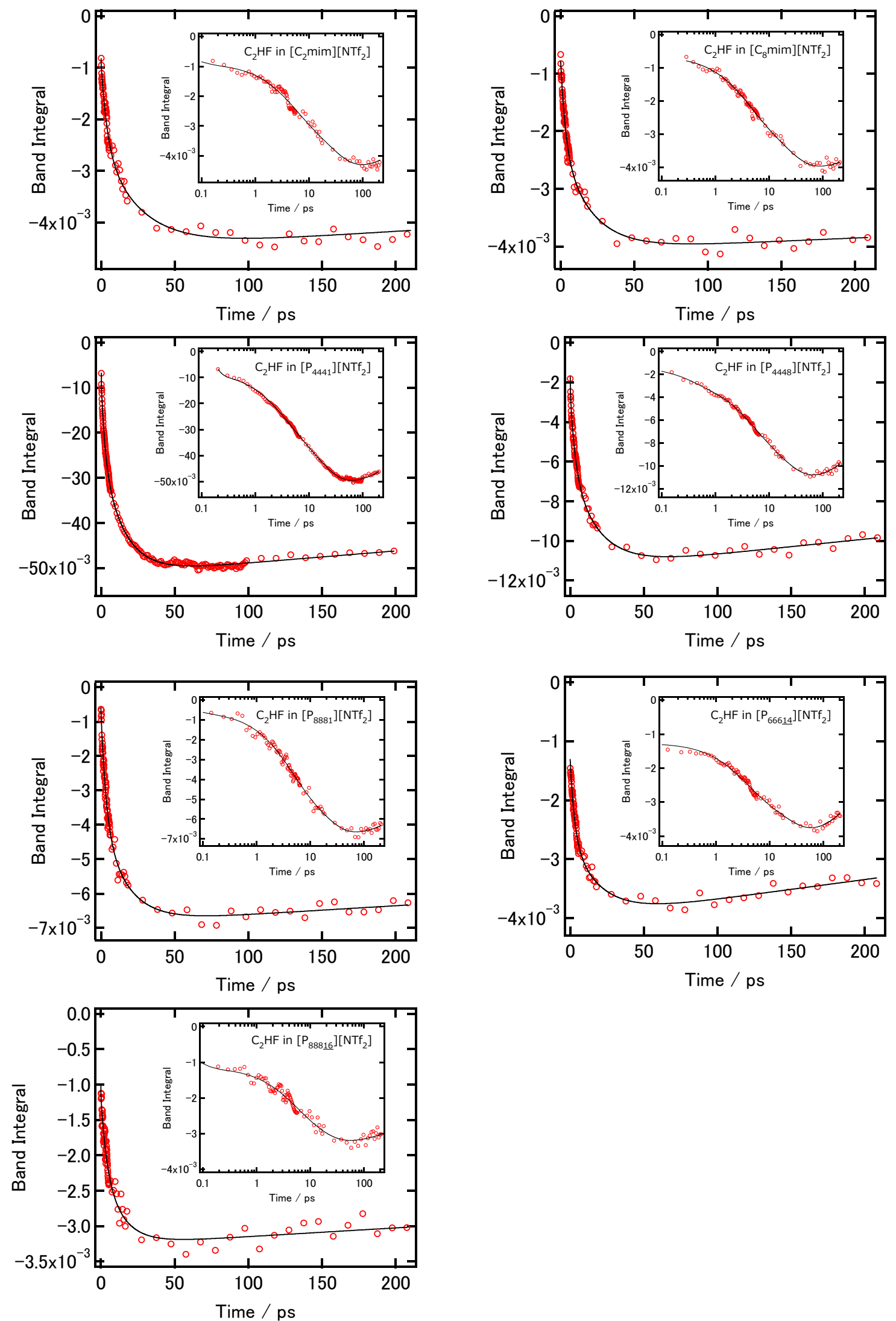
Figure S7(a-2). Time profile of the band integral of IE of $\mathrm{T}^{*}$ for $\mathrm{C}_{4} \mathrm{HF}$ excited at $400 \mathrm{~nm}$. The band integral data are evaluated from the spectral deconvolution analysis. Black lines represent the fitting to Eq (6).
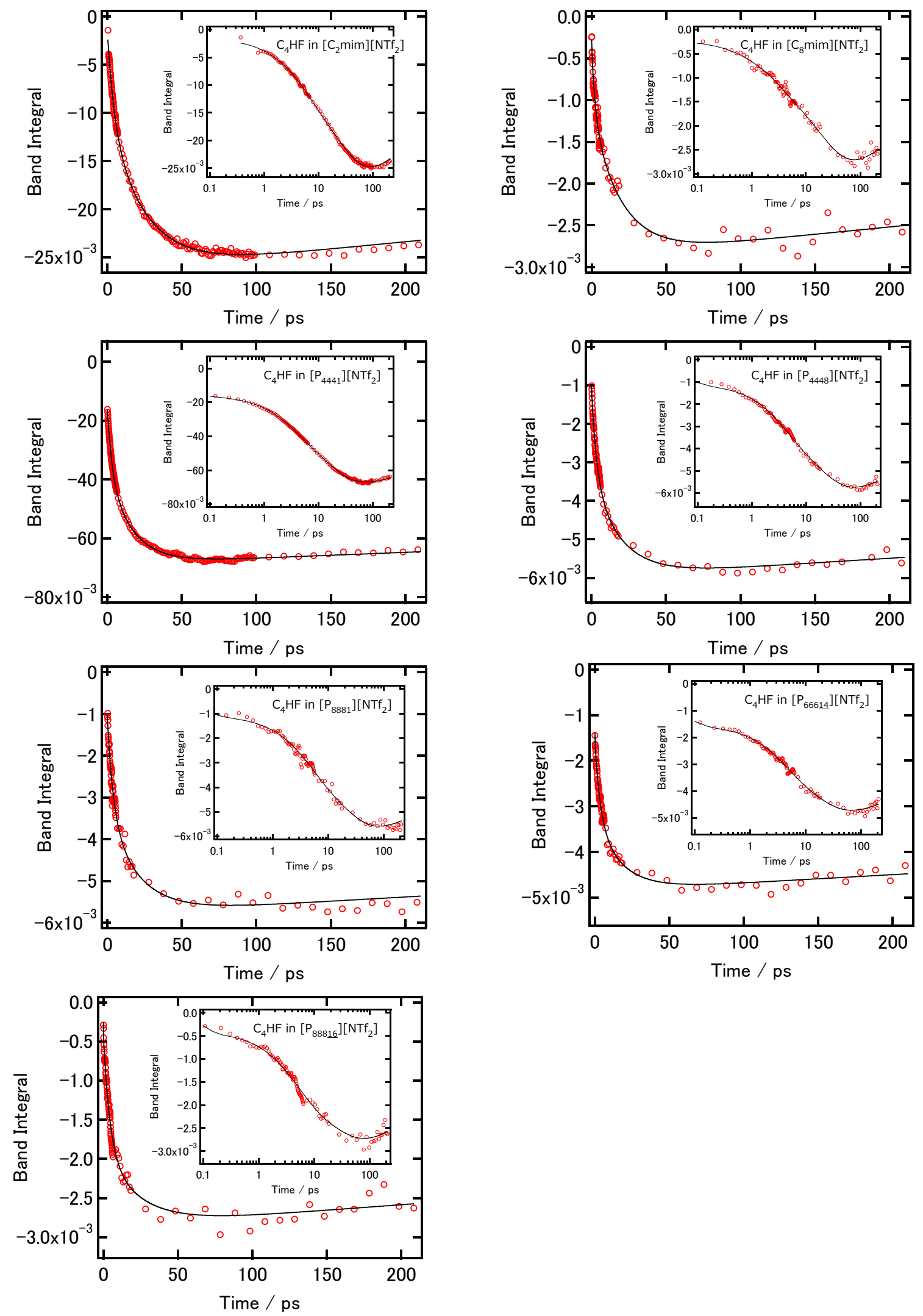
Figure S7(a-3). Time profile of the band integral of IE of $\mathrm{T}^{*}$ for $\mathrm{C}_{8} \mathrm{HF}$ excited at $400 \mathrm{~nm}$. The band integral data are evaluated from the spectral deconvolution analysis. Black lines represent the fitting to Eq (6).
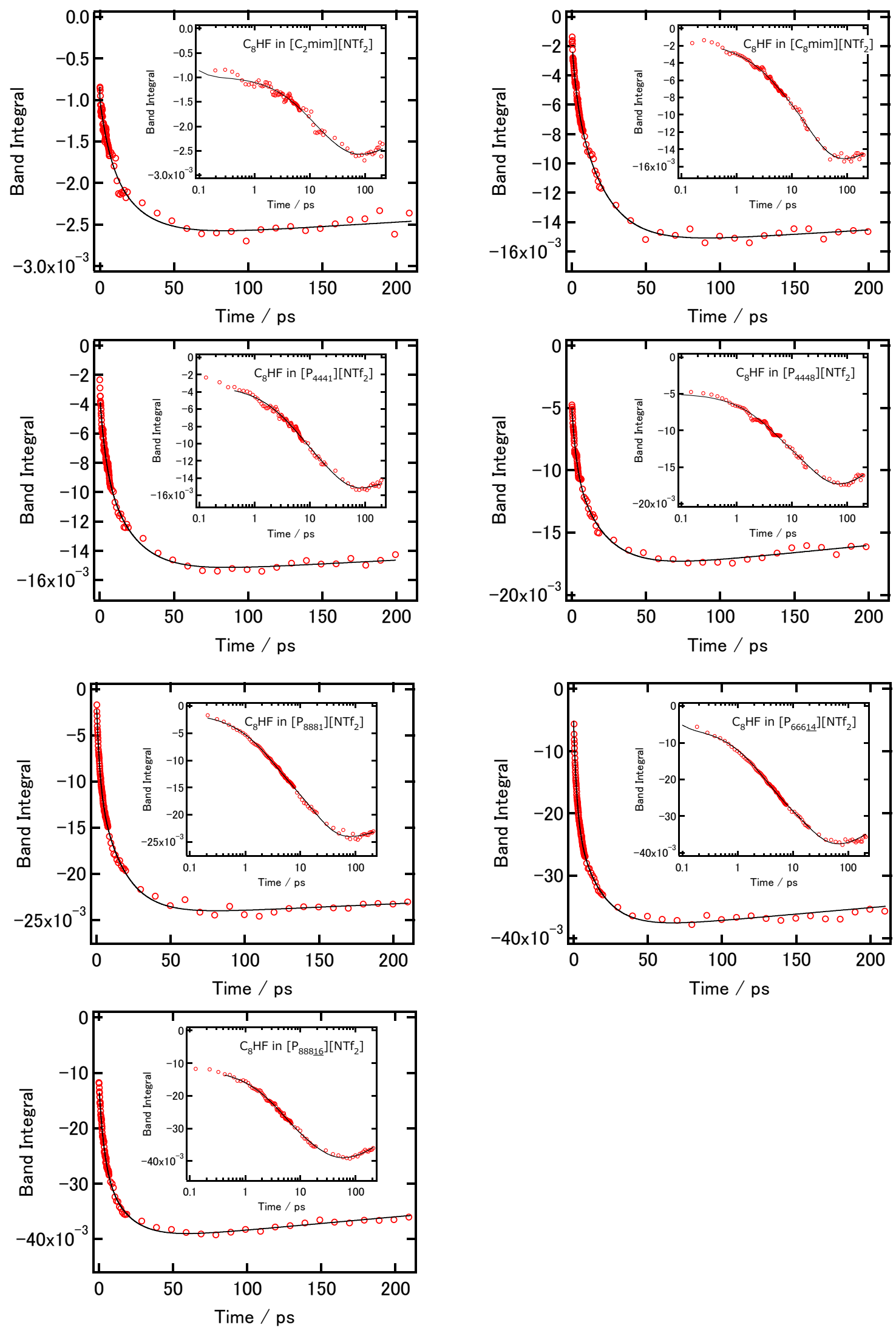
Figure S7(b-1). Time profile of $\Delta \mathrm{OD}$ at the band center of IE of $\mathrm{T}^{*}$ for $\mathrm{C}_{2} \mathrm{HF}$ excited at $400 \mathrm{~nm}$. Black lines represent the fitting to $\mathrm{Eq}(6)$.
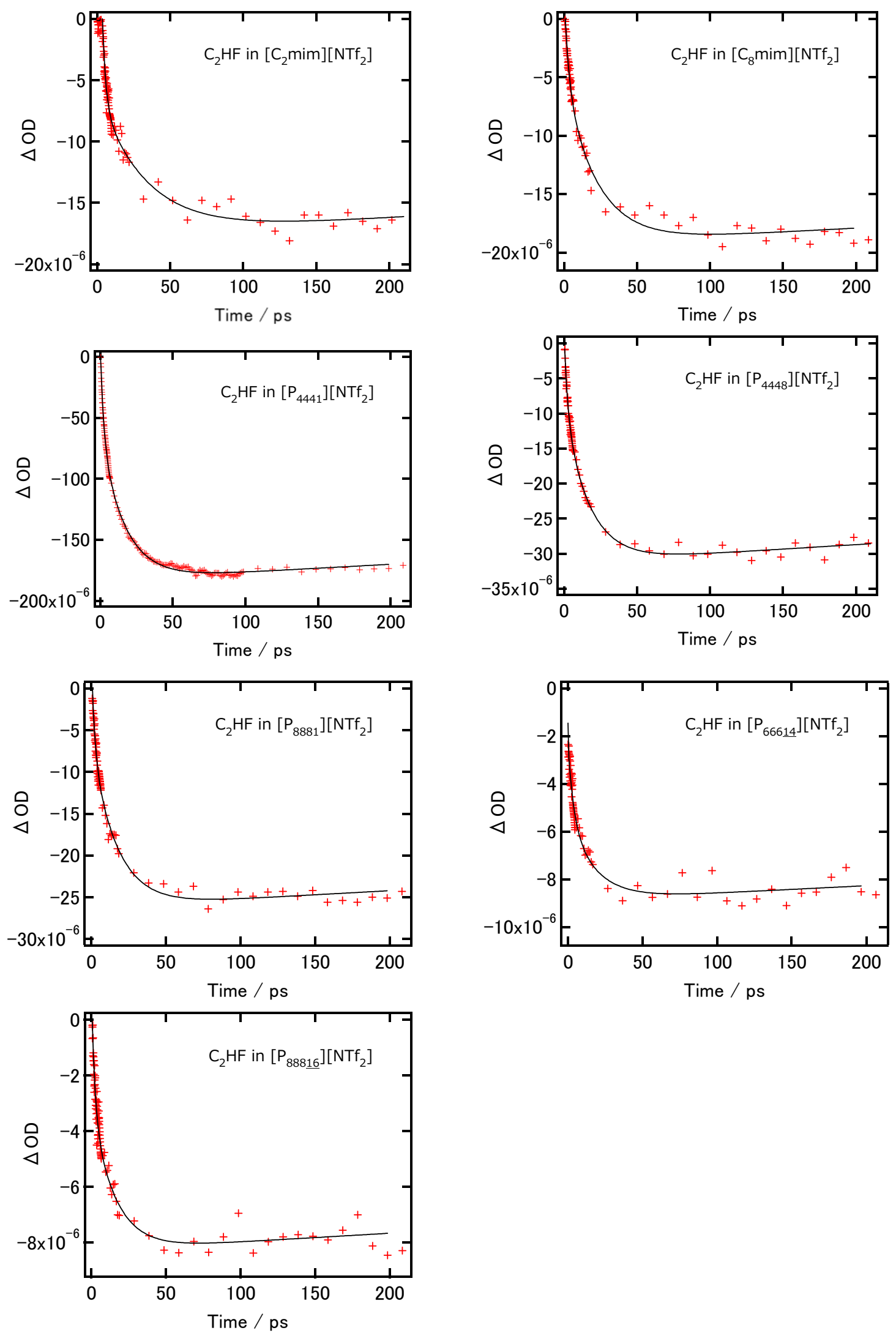
Figure S7(b-2). Time profile of $\Delta \mathrm{OD}$ at the band center of IE of $\mathrm{T}^{*}$ for $\mathrm{C}_{4} \mathrm{HF}$ excited at $400 \mathrm{~nm}$. Black lines represent the fitting to $\mathrm{Eq}(6)$.
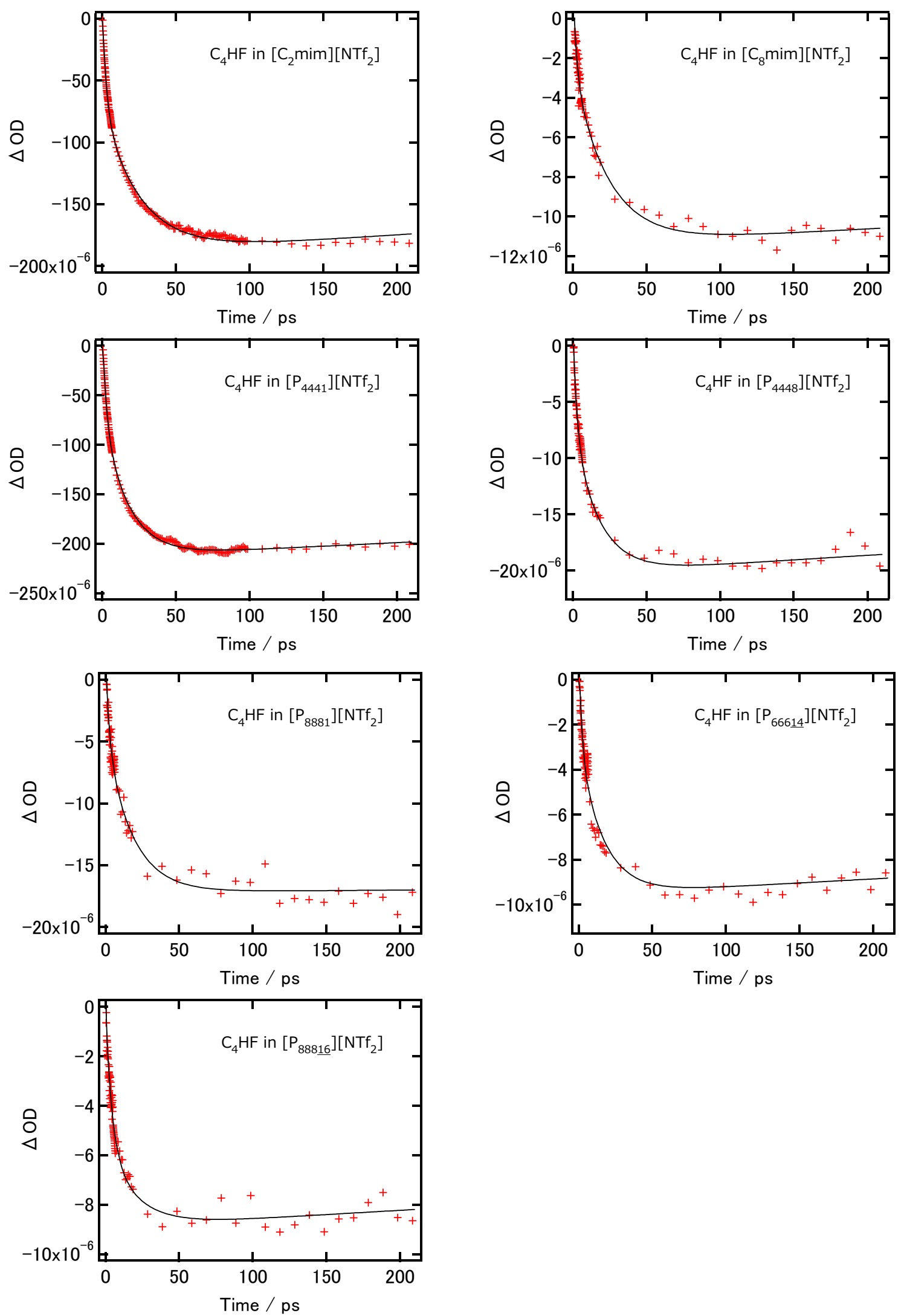
Figure S7(b-3). Time profile of $\Delta \mathrm{OD}$ at the band center of IE of $\mathrm{T}^{*}$ for $\mathrm{C}_{8} \mathrm{HF}$ excited at $400 \mathrm{~nm}$. Black lines represent the fitting to $\mathrm{Eq}(6)$.
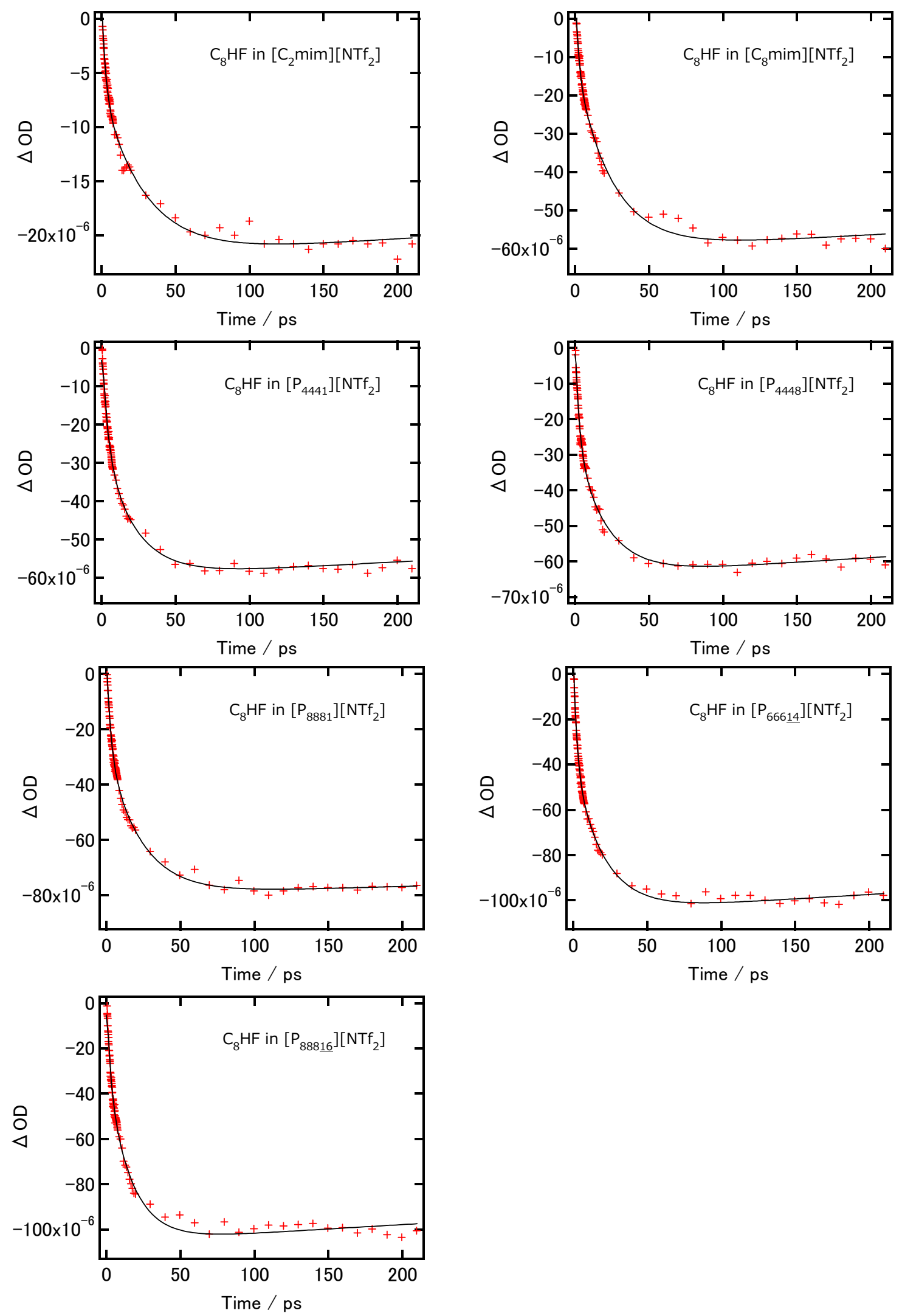
Figure S8. Comparison between $\tau_{\mathrm{ESIPT}}$ estimated by IE of $\mathrm{T}^{*}$ obtained from the band integral (red circles) and from $\triangle \mathrm{OD}$ at the band center (blue diamonds) for (a) $\mathrm{C}_{2} \mathrm{HF}$, (b) $\mathrm{C}_{4} \mathrm{HF}$ and (c) $\mathrm{C}_{8} \mathrm{HF}$. The band integral data are evaluated from the spectral deconvolution analysis. The open markers represent those in the imidazolium-based ILs, and the filled ones in the phosphonium-based ILs. (d) Correlation between $\tau_{\text {EIPT }}$ obtained from the band integral and from the $\Delta \mathrm{OD}$ at the band center.
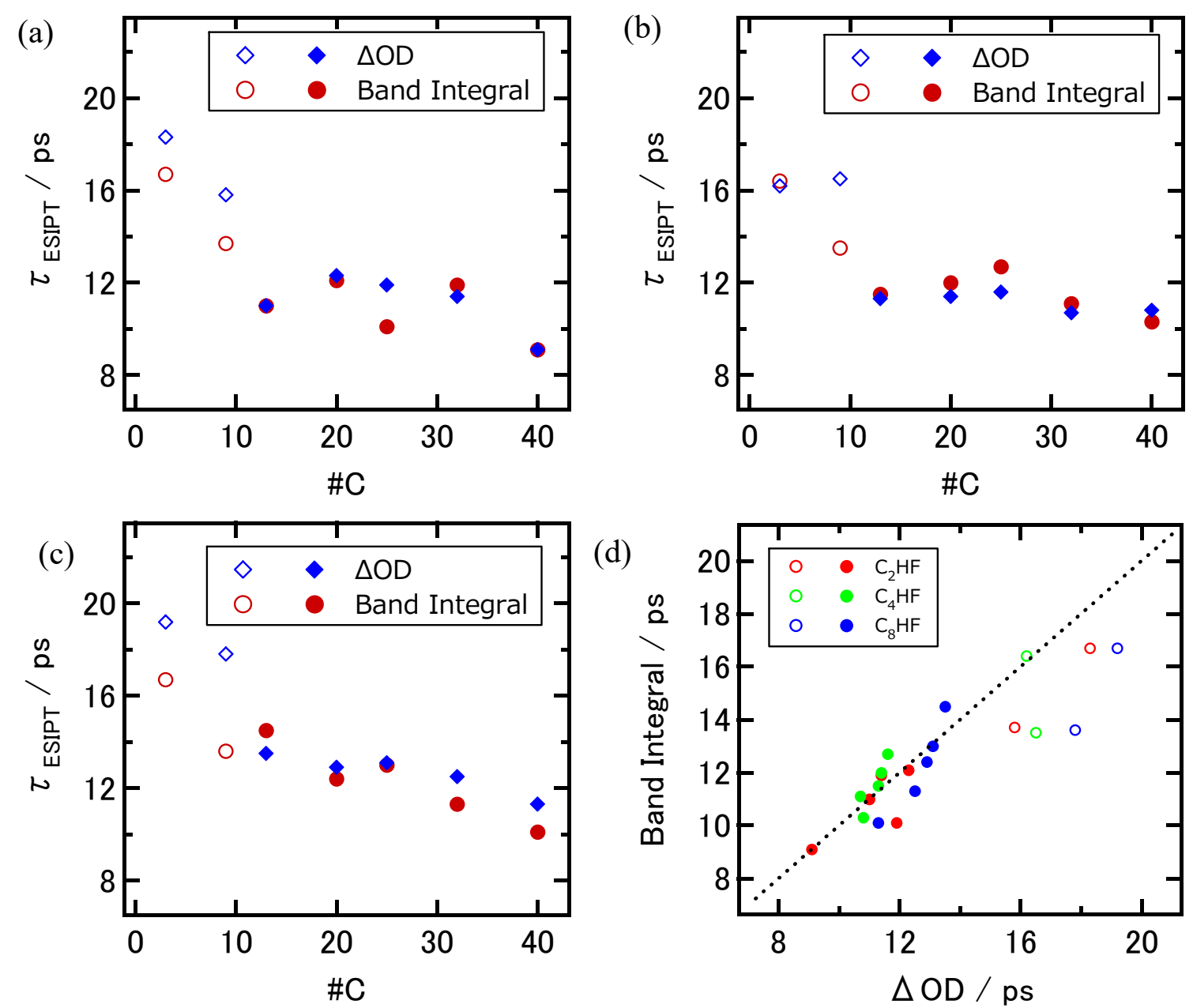

(d)

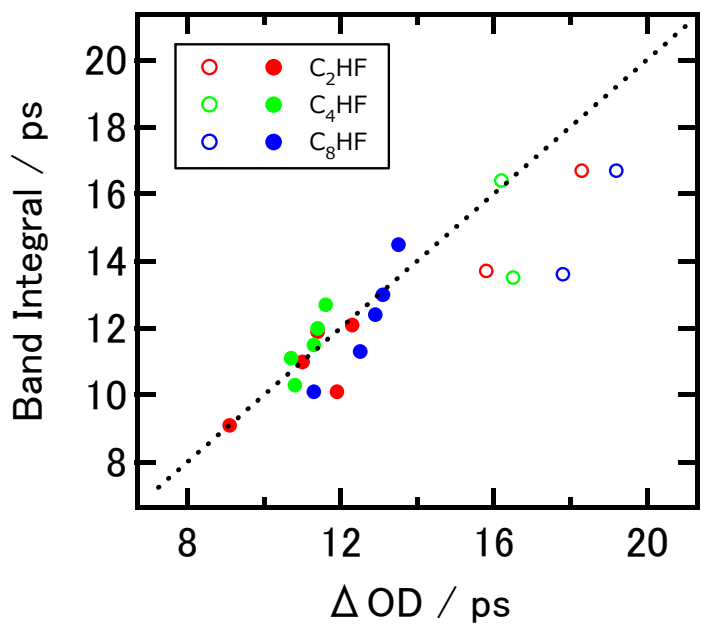


Figure S9(a). Time profile of $\Delta \mathrm{OD}$ at the band center of IE of $\mathrm{T}^{*}$ excited at $360 \mathrm{~nm}$. Black lines represent the fitting to $\mathrm{Eq}(6)$.
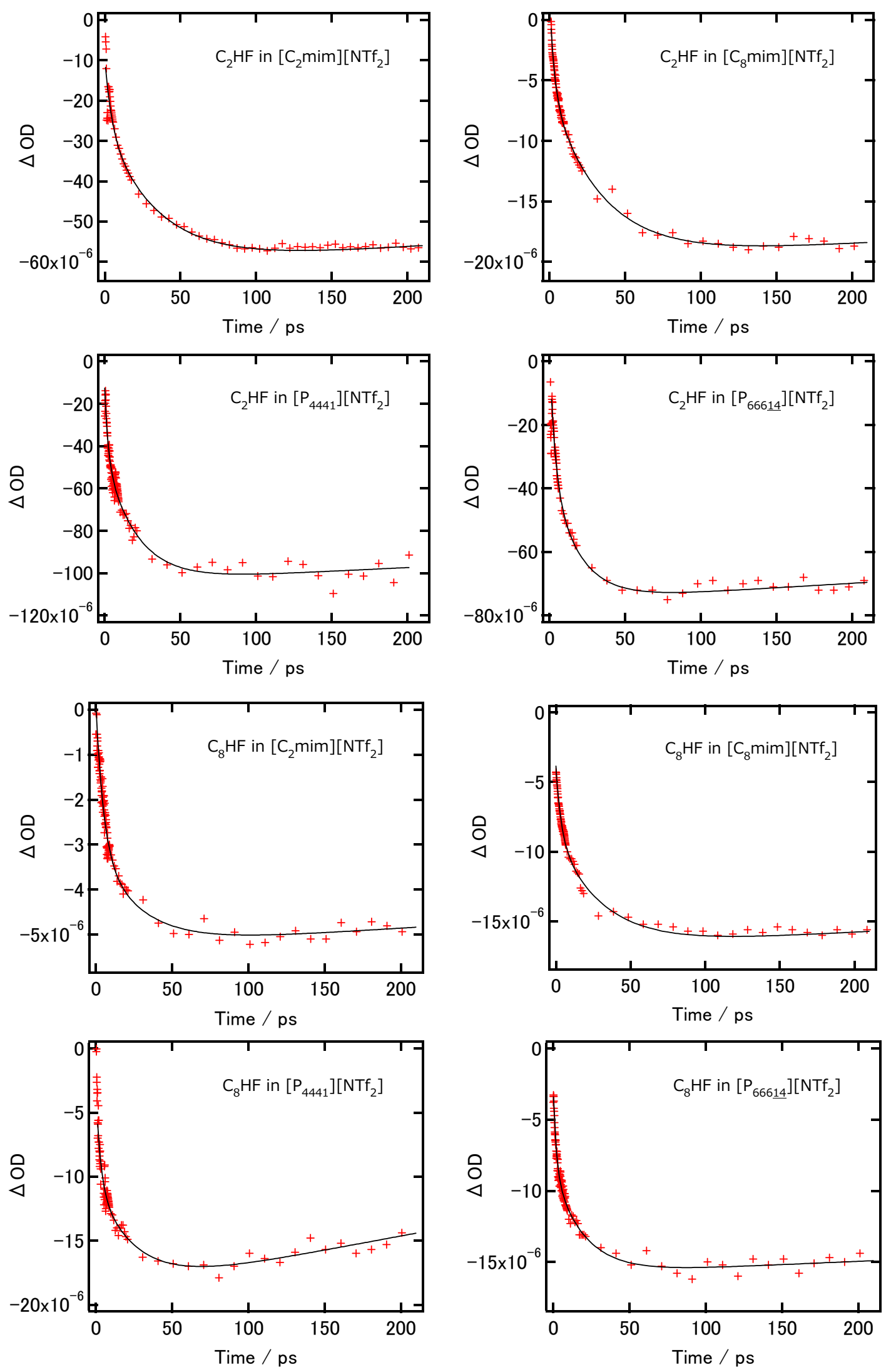
Figure $\mathrm{S} 9(\mathrm{~b})$. Time profile of $\Delta \mathrm{OD}$ at the band center of IE of $\mathrm{T}^{*}$ excited at $450 \mathrm{~nm}$. Black lines represent the fitting to $\mathrm{Eq}(6)$.
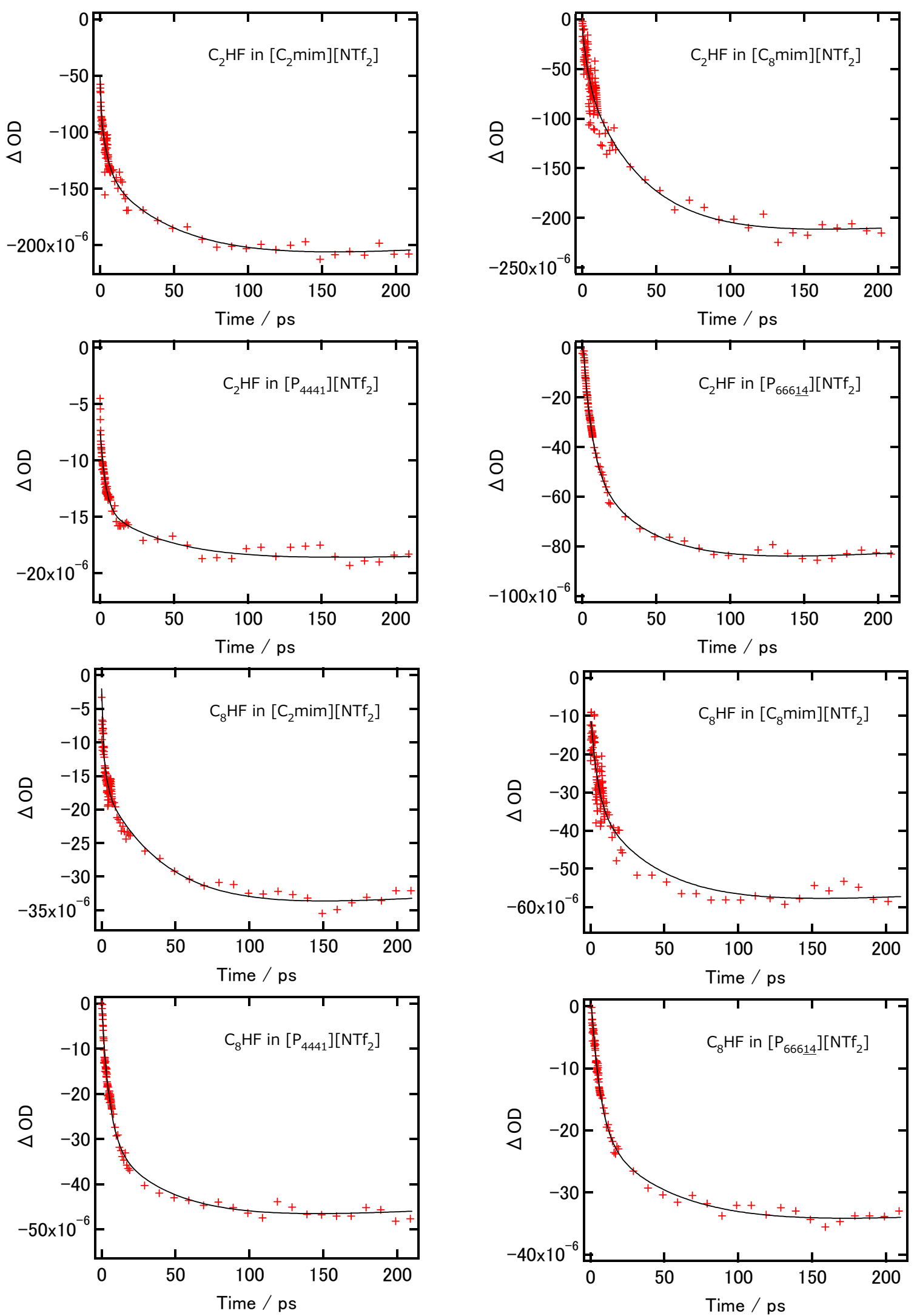
Figure S10. The peak position of the ground-state bleach excited at $450 \mathrm{~nm}$. Black lines represent the fitting to Eq. (8).
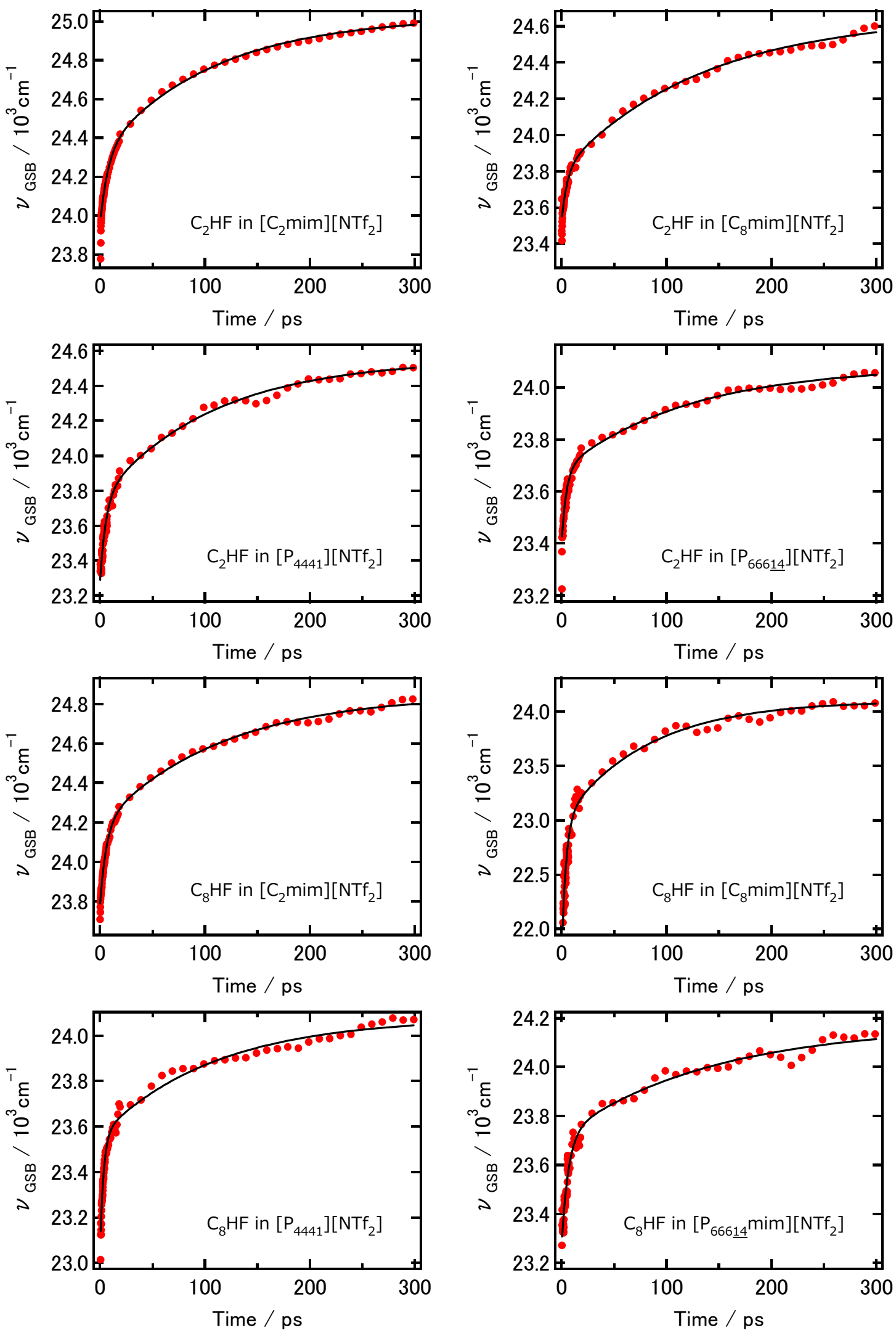


\section{References for supporting information}

(1) Rumble, C. A.; Breffke, J.; Maroncelli, M. Solvation dynamics and proton transfer in diethylaminohydroxyflavone. J. Phys. Chem. B 2017, 121, 630-637

(2) Kimura, Y.; Fukuda, M.; Suda, K.; Terazima, M. Excited state intramolecular proton transfer reaction of 4'-N, N-diethylamino-3-hydroxyflavone and solvation dynamics in room temperature ionic liquids studied by optical Kerr gate fluorescence measurement. J. Phys. Chem. $B$ 2010, $114,11847-11858$

(3) Suda, K.; Terazima, M.; Sato, H.; Kimura, Y. Excitation wavelength dependence of excited state intramolecular proton transfer reaction of 4'-N,N-diethylamino-3-hydroxyflavone in room temperature ionic liquids studied by optical Kerr gate fluorescence measurement J. Phys. Chem. B 2013, 117, 12567-12582

(4) Trariq, M.; Forte, P. A. S.; Gomes, M. F. C.; Lopes, J. N. C.; Rebelo, L. P. N. Densities and refractive indices of imidazolium- and phosphonium-based ionic liquids: Effect of temperature, alkyl chain length, and anion J. Chem. Thermodyn 2009, 41, 790-798

(5) Tsunashima, K.; Sugiya, M. Physical and electrochemical properties of room temperature ionic liquids based quaternary phosphonium cations. Electrochemistry 2007, 75, 734-736

(6) Kimura, Y.; Fukui, T.; Okazoe, S.; Miyabayashi, H.; Endo, T. Photo-excitation dynamics of N,N-diethyl-p-nitroaniline in ionic liquids: Effect of cation alkyl-chain length. J. Mol. Liq. 2019, 289,111128

(7) Kimura, Y.; Hamamoto, T.; Terazima, M. Raman spectroscopic study on the solvation of N, N-dimethyl-p-nitroaniline in room-temperature ionic liquid. J. Phys. Chem. A 2007, 111, 70817089

(8) Kobayashi, A.; Osawa, K.; Terazima, M.; Kimura, Y. Solute-solvent hydrogen-bonding in room temperature ionic liquids studied by Raman spectroscopy. Phys. Chem. Chem. Phys. 2012, $14,13676-13683$ 
(9) Nikam, P.S.; Shirsat, L.N.; Hasan,M. Density and viscosity studies of binary mixtures of acetonitrile with methanol, ethanol, propan-1-ol, propan-2-ol, butan-1-ol, 2-methylpropan-1-ol, and 2-methylpropan-2-ol at (298.15, 303.15, 308.15, and 313.15) K. J. Chem. Eng. Data. 1998, $43,732-737$

(10) Reichardt, C. Solvatochromic dyes as solvent polarity indicators. Chem. Rev. 1994, 94, $2319-2358$

(11) Reichardt, C. Solvents and solvent effects in organic chemistry. Wiley, Third edition, 2003 